\title{
Clonal Distribution of Invasive Pneumococcal Isolates from Children and Selected Adults in the United States Prior to 7-Valent Conjugate Vaccine Introduction
}

\author{
Robert E. Gertz, Jr., ${ }^{1}$ M. Catherine McEllistrem, ${ }^{2}$ David J. Boxrud, ${ }^{3}$ Zhongya $\mathrm{Li},{ }^{1}$ Varja Sakota, ${ }^{1}$ \\ Terry A. Thompson, ${ }^{1}$ Richard R. Facklam, ${ }^{1}$ John M. Besser, ${ }^{3}$ Lee H. Harrison, ${ }^{4}$ \\ Cynthia G. Whitney, ${ }^{1}$ and Bernard Beall ${ }^{1 *}$ \\ Division of Bacterial and Mycotic Diseases, Centers for Disease Control and Prevention, Atlanta, Georgia ${ }^{1}$; Division of \\ Infectious Diseases, University of Pittsburgh, Pittsburgh, Pennsylvania ${ }^{2}$; Acute Disease Epidemiology Section and \\ Division of Public Health Laboratories, Minnesota Department of Health, Minneapolis, Minnesota ${ }^{3}$; \\ and Department of International Health, Johns Hopkins University Bloomberg
} School of Public Health, Baltimore, Maryland ${ }^{4}$

Received 7 February 2003/Returned for modification 7 April 2003/Accepted 17 April 2003

\begin{abstract}
The seven-valent pneumococcal conjugated polysaccharide vaccine PC7V was licensed for use among children in 2000. Since 90 serotypes of pneumococci exist, an increase in nonvaccine serotypes could occur through immune selection for capsular type switching. Eleven hundred sixty-eight invasive isolates (24 serotypes), recovered primarily from pediatric patients $(855$ isolates $=73 \%)$ and 22 reference strains of known multilocus sequence types (STs) were subjected to macrorestriction profiling (pulsed-field gel electrophoresis [PFGE]). The correlation of 187 ST results (including 49 newly discovered STs) with the PFGE data assigned 1,042 (89.2\%) study isolates to 46 defined clonal complexes or genetic lineages based on related multilocus STs (BURST). Seventeen clonal complexes were represented by 2 to 10 related allelic profiles (STs), while 33 lineages (including reference strains) consisted of single STs with 4 or fewer allelic identities to other STs found in the study. Expansion of the BURST analysis to a global analysis of all known pneumococcal STs (as of 27 November 2002) reduced the number of single ST lineages from 33 to 8 , and the number of multi-ST clonal complexes was reduced from 17 to 13 . In this work, we established the basic genetic structure within individual serotypes prior to $\mathrm{PC7V}$ use. The resultant database will be useful for detecting potential selective effects of this vaccine in postvaccine surveillance.
\end{abstract}

Accurate molecular surveillance is necessary to understand the effects of selective pressures on the population genetics of pneumococci. This is especially true at the present time with a newly introduced seven-valent conjugate vaccine (PCV7) that is projected to be highly effective in decreasing the invasive pneumococcal disease burden. To monitor potential selective effects of this vaccine on invasive pneumococcal strain distribution, precise genetic analysis is required to provide a baseline of prevaccine strain-serotype associations.

Pulsed-field gel electrophoresis (PFGE) of chromosomal macrorestriction digests and multilocus sequence typing (MLST) are complementary techniques that are well suited for the analysis of large numbers of isolates. PFGE is ideal for the rapid clustering of genetically related isolates. MLST can be used to assign nonsubjective and electronically portable clone identifiers to clusters of related isolates.

We have used PFGE and MLST to clearly delineate strain distribution within a large set of population-based invasive pneumococcal isolates recovered prior to the use of PCV7. Isolates recovered from adults with selected serotypes that are rare in children, or in which reduced antibiotic susceptibility had been previously reported (34), were also included in this analysis.

\footnotetext{
* Corresponding author. Mailing address: CDC Respiratory Diseases Branch, Mailstop C02, 1600 Clifton Rd., N.E., Atlanta, GA 30333. Phone: (404) 639-1237. Fax: (404) 639-3123. E-mail: bbeall @cdc.gov.
}

\section{MATERIALS AND METHODS}

Isolates. Eleven hundred sixty-eight sterile-site isolates recovered during 1999 from individuals within the United States were analyzed. Isolates were collected through Centers for Disease Control and Prevention (CDC) Active Bacterial Core Surveillance (ABCs); methods for case identification and isolate collection have been previously described (34). The surveillance areas included California (San Francisco County), Connecticut, Georgia (20-county Atlanta area), Maryland (6-county Baltimore area), Minnesota (7-county twin city area), New York (7-county Rochester and 8-county Albany areas), Oregon (3-county Portland area), and Tennessee (11 urban counties). The surveillance areas represented about 18.5 million persons at the beginning of 1999, and this was increased to about 19.8 million by approximately mid-1999 because of the increase from 5 to 11 Tennessee counties. All isolates were serotyped and subjected to antibiotic susceptibility testing as previously described (16). Patterns of resistance to chloramphenicol, trimethoprim-sulfamethoxazole, erythromycin, clindamycin, penicillin, cefotaxime, tetracycline, and levofloxacin are presented with MLST results of selected isolates (see Fig. 1 to 13). Within each serotype, isolates included 63.5 to $100 \%$ (median of about $86 \%=854$ of 998 isolates) of available ABCs isolates recovered from children less than 5 years of age during 1999. Seventy-one to 208 pediatric isolates for each of the PCV7 serotypes $(4,6 \mathrm{~B}, 9 \mathrm{~V}, 14,18 \mathrm{C}, 19 \mathrm{~F}$, and $23 \mathrm{~F}$ ) were included. In addition, non-PCV7 serotypes were analyzed, such that isolates of serotypes $1,3,5,6 \mathrm{~A}, 7 \mathrm{~B}, 7 \mathrm{~F}, 9 \mathrm{~N}, 11 \mathrm{~A}, 12 \mathrm{~F}, 15 \mathrm{~B}, 15 \mathrm{C}, 16 \mathrm{~F}, 19 \mathrm{~A}, 22 \mathrm{~F}$, $33 \mathrm{~F}, 35 \mathrm{~B}$, and 38 were included. Three hundred fourteen invasive isolates from adult patients were included. These included selected serotypes that have displayed decreased antibiotic susceptibility in recent years (34) and certain serotypes that are rare in children. In addition, characterized reference strains recovered in the United States and other countries were included for PFGE analysis $(2,14,15,16-21,23,26-28,30,32)(1)$. All available isolates exhibiting unusual genotype-serotype or serotype-penicillin MIC combinations were subjected to an appropriate reanalysis that included one or more of the tests for serotyping, PFGE, MLST, and MIC determination. 
TABLE 1. Pneumococcal strains of known STs used as PFGE references

\begin{tabular}{|c|c|c|c|}
\hline $\begin{array}{l}\text { PFGE-MLST } \\
\text { reference strain }\end{array}$ & Description & ST & Reference(s) \\
\hline Spain $23 \mathrm{~F}-1$ & Widely disseminated clone first identified in Spain, has been recovered before 1991 & ST81 & 23,26 \\
\hline Spain $^{6 \mathrm{~B}}-2$ & Widely disseminated, multiresistant strain first identified in Spain during 1980s & ST90 & 23,27 \\
\hline Spain $^{9 \mathrm{~V}}-3$ & Multiresistant strain first identified in France and Spain prior to 1991 & ST156 & $2,18,23$ \\
\hline Tennessee ${ }^{23 \mathrm{~F}}-4$ & Multiresistant, highly cephalosporin-resistant major clone found throughout United States & ST37 & 21,23 \\
\hline Spain $^{14}-5$ & Multiresistant strain first identified in Spain during 1990-1994 & ST18 & 2,23 \\
\hline Hungary ${ }^{19 A}-6$ & $\begin{array}{l}\text { Multiresistant strain first isolated in Hungary, (prior to 1991) and also recovered in Czech } \\
\text { Republic and Slovakia }\end{array}$ & ST226 & $6,10,23$ \\
\hline South Africa ${ }^{19 \mathrm{~A}}-7$ & $\begin{array}{l}\text { Intermediately penicillin-resistant, trimethoprim-sulfamethoxazole-resistant strain } \\
\text { representing a predominant South African clone }\end{array}$ & ST75 & 23,32 \\
\hline South Africa ${ }^{6 \mathrm{~B}}-8$ & Intermediately penicillin-resistant strain representing a predominant South African clone & ST185 & 23,32 \\
\hline England $^{14}-9$ & Erythromycin-resistant strain first characterized in United Kingdom & ST9 & 17,23 \\
\hline $\mathrm{CSR}^{14}-10$ & Multi-resistant strain recovered in Slovakia and Czech Republic prior to 1995 & ST20 & 14,23 \\
\hline Czech Republic ${ }^{19 \mathrm{~A}}-11$ & Multiresistant strain recovered in Slovakia and Czech Republic prior to 1995 & ST175 & 14,23 \\
\hline Finland ${ }^{6 \mathrm{~B}}-12$ & Multiresistant strain recovered in Finland prior to 1992 & ST270 & 23,31 \\
\hline South Africa ${ }^{19 \mathrm{~A}}-13$ & Multiresistant strain representing a predominant South African clone & ST41 & 23,32 \\
\hline Taiwan ${ }^{19 F}-14$ & Major Taiwan multirestant clone & ST236 & 23,30 \\
\hline Taiwan $^{23 \mathrm{~F}}-15$ & Major Taiwan multirestant clone & ST242 & 23,30 \\
\hline Poland ${ }^{23 F}-16$ & Multiresistant major clone in Poland & ST173 & 23,28 \\
\hline Maryland $^{6 \mathrm{~B}}-17$ & Multiresistant invasive PFGE type D, recovered in United States during 1997 & ST384 & 16 \\
\hline Tennessee $^{14}-18$ & $\begin{array}{l}\text { Multiresistant invasive PFGE type H strain recovered in United States during 1997; ST67 } \\
\text { also reported at www.mlst.net for Taiwan multiresistant type } 14 \text { strain }\end{array}$ & ST67 & 16 \\
\hline Chile-19F V1 & $\begin{array}{l}\text { Major type 19F PFGE type V1 strain recovered between } 1994 \text { and } 1999 \text { from } \\
\text { asymptomatic children in Chile }\end{array}$ & ST646 & 15 \\
\hline CT-19F G1 & $\begin{array}{l}\text { Multiresistant type 19F invasive PFGE type G, recovered in United States during 1997; } \\
\text { ST271 also recorded at www.mlst.net from Arizona during } 1994 \text { and Korea; SLV of } \\
\text { Taiwan }{ }^{19 F}-14\end{array}$ & ST271 & 16 \\
\hline San Francisco-19F 10753 & $\begin{array}{l}\text { Multiresistant (including levofloxacin resistance) invasive type 19F isolate recovered from } \\
\text { adult during } 1997\end{array}$ & ST650 & $\begin{array}{l}\text { B. Beall (unpub- } \\
\text { lished data) }\end{array}$ \\
\hline New Hampshire (NT) ${ }^{a}$ & $\begin{array}{l}\text { Nontypeable strain causing large conjunctivitis outbreak among students at a New } \\
\text { Hampshire college }\end{array}$ & ST448 & 19 \\
\hline
\end{tabular}

${ }^{a}$ NT, nontypeable.

Rationale for isolate selection. The purpose of this work was to genetically profile all available year 1999 pediatric invasive pneumococcal isolates, with the intentional exception of serotype 14 (the most common type), for which about $63 \%$ of the isolates $(n=208)$ were analyzed (Table 2$)$. We reasoned that this number of isolates should adequately reflect the genetic structure of serotype 14 . Additional isolates from older individuals representing common invasive serotypes not well represented among the pediatric isolates $(1,3,12 \mathrm{~F}$, and $22 \mathrm{~F})$ and selected serotypes recently associated with decreased antibiotic susceptibility (7F, 9N. 11A, 19A, and 35B) (3) were also included. All PCV7 serotypes (4, 6B, $9 \mathrm{~V}, 14,18 \mathrm{C}, 19 \mathrm{~F}$, and $23 \mathrm{~F}$ ) and serotype $6 \mathrm{~A}$ were well represented among the available pediatric isolates (64 to 208 isolates) (see Fig. 1 to 13; Table 2).

PFGE and MLST. PFGE was performed as described previously (22). MLST was performed as previously described (8) with the modification of using the slightly longer primers below, which improved the consistency of our results. Sequencing was performed with an ABI 377 apparatus. The following primers were used: AROE-UP1, GCCTTTGAGGCGACAGCTACCAACG; AROEDN1, TGCAGTTCA(G/A)AAACAT(A/T)TTCTAAAG; GDH-UP1, ATGGA CAAACCAGC(G/A/T/C)AG(C/T)TTCACAAAAG; GDH-DN1, GCTTGA GGTCCCAT(G/A)CT(G/A/T/C)CCAGCTTTAT; GKI-UP1, GGCATTGG AATGGGATCACCAGGTGT; GKI-DN1, TCTCCCGCAGCTGACACACC ACCA; RECP-UP1, GCCAACTCAGGTCATCCAGGTGTGG; RECP-DN1, TGCAACCGTAGCATTGTAACGGTCA; SPI-UP1，TTATTCCTCCTGATT CTGTCATTACTA; SPI-DN1, GTGATTGGCCAGAAGCGGAATTTAGCT; XPT-UP1, TTATTAGAAGAGCGCATCCTCAAGGA; XPT-DN1, AGATCT GCCTCCTTAAATACGAC(T/C)TG; DDL-UP1，TGC(C/T)CAAGTTCCTT ATGTGGCTATCG; DDL-DN1, CACTGGGT(G/A)AAACC(A/T)GGCAT (A/G)GTATTG.

PFGE analysis. The Bionumerics software (Applied-Maths, Kortrijk, Belgium) was used to make UPGMA (unweighted pair group method with arithmetic means) dendrograms of fragment patterns with the dice coefficient. The dice similarity coefficient was used with optimization and position tolerance settings of 1.0 and $1.5 \%$, respectively. PFGE-based clusters were defined as isolates with $\geq 80 \%$ genetic relatedness on the dendrogram.

MLST analysis. MLST alleles from www.mlst.net were downloaded for convenient FASTA screening with the Wisconsin version 10.2 package. New alleles were screened at www.mlst.net to ensure that they were still not included in the pneumococcal MLST database. For the 28 new alleles discovered during this work, sequence traces of both strands were electronically submitted to the pneumococcal database curator for approval prior to their inclusion in the database. Clonal complex (CC) or lineage assignment (BURST) was carried out with software downloaded from http://outbreak.ceid.ox.ac.uk/software.shtml and was used as directed, requiring the parameter of five identical targets with at least one other isolate in the set. MLST data were also used for UPGMA-generated dendrograms with the software downloaded from this web site.

\section{RESULTS}

Correlation of PFGE and MLST data. Eleven hundred sixtyeight invasive isolates (24 serotypes), recovered primarily from pediatric patients $(854$ isolates $=73.1 \%)$, and 22 reference strains were subjected to PFGE. A total of 165 study isolates and 4 reference strains from various sources (CT PFGE G1, Chile V1, New Hampshire NT, and San Francisco 10753) were subjected to MLST in this study (Table 1). In addition, 18 internationally disseminated multiresistant reference strains for which MLST results were either published (23) or communicated by Brian Spratt were included in the analysis (Table 1).

The PFGE-MLST associations reported here are documented by serotype (Fig. 1 to 13 and Table 2). All of the serotypes that included 20 or more isolates were represented in at least six of the eight states included in the surveillance (data not shown). All MLST results are superimposed on the PFGE dendrograms shown in Fig. 1 to 13.

We attempted to relate the PFGE clusters (individual patterns differing by a UPGMA dice coefficient of $\leq 20 \%$ ) within specific serotypes with specific allelic profiles or clusters of related allelic profiles by applying MLST to representative isolates. When we observed similar PFGE profiles shared by 
TABLE 2. Clonal complex or single ST assignment of 1,042 study isolates based on PFGE and MLST data

\begin{tabular}{|c|c|c|c|c|c|c|}
\hline \multirow[t]{2}{*}{ Serotype } & \multicolumn{2}{|c|}{$\begin{array}{l}\text { No. of isolates stud- } \\
\text { ied within serotype } \\
\text { recovered from indi- } \\
\text { viduals ( } \% \text { of total } \\
\text { pediatric surveillance) }\end{array}$} & \multirow[t]{2}{*}{$\begin{array}{l}\text { STs of same clonal complex or single ST lineage } \\
\text { within this serotype in study isolates }{ }^{a} \text { (serotypes listed at } \\
\text { www.mlst.net for STs deposited prior to new ones } \\
\text { discovered here) }\end{array}$} & \multirow[t]{2}{*}{$\begin{array}{l}\text { Estimated } \% \text { of } \\
\text { serotype within } \\
\text { ST lineage } \\
\text { (no. of isolates) }^{b}\end{array}$} & \multirow[t]{2}{*}{$\begin{array}{c}\% \beta \text {-Lactam } \\
\text { nonsusceptible } \\
\text { within indicated } \\
\text { ST lineage } \\
\text { (no. of isolates) }\end{array}$} & \multirow[t]{2}{*}{$\begin{array}{l}\% \text { Nonsusceptible } \\
\text { to other antibiotics } \\
\text { within indicated } \\
\text { ST lineage } \\
\text { (no. of isolates) }\end{array}$} \\
\hline & $<5 \mathrm{yr}$ & $\geq 5 \mathrm{yr}$ & & & & \\
\hline \multirow{2}{*}{1} & $6(75)$ & 15 & ST227 (1) & $95(20)$ & 0 & 0 \\
\hline & & & ST217 (1) & $5(1)$ & 0 & $100(1)$ \\
\hline 3 & $6(100)$ & 50 & ST180 (3) & $96.4(54)$ & 0 & $7.4(4)$ \\
\hline \multirow[t]{3}{*}{4} & $71(100)$ & 0 & ST695 & $71.8(51)$ & 0 & $3.3(1)$ \\
\hline & & & ST205 (4) & $11.3(8)$ & 0 & $3.4(1)$ \\
\hline & & & ST244 (4) & $2.8(2)$ & 0 & 0 \\
\hline 5 & $1(100)$ & 0 & ST653 & $100(1)$ & 0 & $100(1)$ \\
\hline \multirow[t]{6}{*}{$6 \mathrm{~A}$} & $57(100)$ & 1 & ST376 (6A) & $32.8(19)$ & $100(19)$ & $100(19)$ \\
\hline & & & ST460 (6A) & $22.4(13)$ & 0 & $23(3)$ \\
\hline & & & ST473 (6A) + ST647 & $8.6(5)$ & $40(2)$ & $40(2)$ \\
\hline & & & ST395 (6A) & $3.4(2)$ & $100(2)$ & $100(2)$ \\
\hline & & & ST660 & $1.7(1)$ & $100(1)$ & $100(1)$ \\
\hline & & & ST90 (6B) & $1.7(1)$ & $100(1)$ & $100(1)$ \\
\hline \multirow[t]{6}{*}{$6 \mathrm{~B}$} & $103(95)$ & 0 & ST138 (6B) + ST639 & $23.3(24)$ & $4.2(1)$ & 0 \\
\hline & & & ST146 (6B) + ST624 + ST640 + ST641 & $21.4(22)$ & $18.2(4)$ & $45(10)$ \\
\hline & & & ST90 (6B) & $12.6(13)$ & $100(13)$ & $100(13)$ \\
\hline & & & ST384 (6B) & $5.8(6)$ & $100(6)$ & $100(6)$ \\
\hline & & & ST644 & $2.9(3)$ & 0 & $67(2)$ \\
\hline & & & ST185 (6B) & $1(1)$ & 0 & $100(1)$ \\
\hline $7 \mathrm{~B}$ & 0 & 1 & ST664 & $100(1)$ & $100(1)$ & $100(1)$ \\
\hline \multirow[t]{2}{*}{$7 \mathrm{~F}$} & $7(87.5)$ & 24 & ST191 (7F) & $93.5(29)$ & 0 & 0 \\
\hline & & & ST218 (12F) & $3.2(1)$ & 0 & 0 \\
\hline $9 \mathrm{~N}$ & $2(100)$ & 11 & ST631 + ST632 & $84.6(11)$ & 0 & $18.2(2)$ \\
\hline $9 \mathrm{~V}$ & $72(100)$ & 26 & ST156 $(9 \mathrm{~V}, 14,19 \mathrm{~F})+\mathrm{ST} 642+\mathrm{ST} 643$ & $91.8(90)$ & $63.3(62)$ & $46.9(46)$ \\
\hline $11 \mathrm{~A}$ & $1(100)$ & 24 & ST62 (11A) + ST630 & $88(22)$ & 0 & $4.5(1)$ \\
\hline $12 \mathrm{~F}$ & $11(83.9)$ & 49 & $\mathrm{ST} 218(12 \mathrm{~F})+\mathrm{ST} 220(12 \mathrm{~F})$ & $98.3(59)$ & 0 & $13.5(8)$ \\
\hline \multirow[t]{7}{*}{14} & $208(63.5)$ & 0 & ST9 (14) + ST13 (14) + ST16 (14) + ST622+ ST623 & $46.2(96)$ & $81.2(78)$ & $97.9(94)$ \\
\hline & & & ST124 (14) + ST656 & $3.8(78)$ & 0 & $3.8(3)$ \\
\hline & & & ST671 & $2.4(5)$ & $100(5)$ & $100(5)$ \\
\hline & & & ST67 (14) & $1.9(4)$ & $100(4)$ & $100(4)$ \\
\hline & & & ST200 (14) + ST667 & $1.0(2)$ & 0 & $50(1)$ \\
\hline & & & ST633 & $0.5(1)$ & $100(1)$ & $100(1)$ \\
\hline & & & ST18 (14) & $0.5(1)$ & $100(1)$ & $100(1)$ \\
\hline $15 \mathrm{~B}+15 \mathrm{C}$ & $6(85.7)$ & 0 & ST199 (15B; 19A, 19) & $100(6)$ & 0 & 0 \\
\hline $16 \mathrm{~F}$ & $1(100)$ & 1 & ST570 & $50(1)$ & 0 & 0 \\
\hline & & & ST659 & $50(1)$ & 0 & 0 \\
\hline 18C (also 3 type & $93(100)$ & 0 & ST113 $(18 \mathrm{C}, 18 \mathrm{~B})+\mathrm{ST} 637+\mathrm{ST} 638+\mathrm{ST} 648$ & $79.6(74)$ & $1.3(1)$ & $2.7(2)$ \\
\hline $18 \mathrm{~B}$ and $118 \mathrm{~F})$ & & & ST496 $(18 \mathrm{C})$ & $8.6(8)$ & 0 & 0 \\
\hline & & & ST666 & $1.1(1)$ & 0 & $100(1)$ \\
\hline & & & ST632 & $1.1(1)$ & 0 & 0 \\
\hline $19 \mathrm{~A}$ & $18(90)$ & 64 & ST199 (19A, 15B) + ST645 & $7.2(59)$ & $67.8(40)$ & $54.2(32)$ \\
\hline & & & ST172 (19A) + ST663 & $2.4(2)$ & $100(2)$ & $50(1)$ \\
\hline & & & ST665 & $1.2(1)$ & 0 & 0 \\
\hline & & & ST485 & $1.2(1)$ & 0 & 0 \\
\hline $19 \mathrm{~F}$ & $110(94.8)$ & 0 & $\begin{array}{l}\text { ST236 (19F) }+ \text { ST271 + (19F) }+ \text { ST635 + ST651 + } \\
\text { ST652 }\end{array}$ & $20.9(23)$ & $100(23)$ & $100(23)$ \\
\hline & & & ST25 (19F) + ST654 & $20(22)$ & $5(1)$ & $10(2)$ \\
\hline & & & ST81 (23F, 19F, 19A, 9V, 14) & $11.8(13)$ & 100 (13) & $100(13)$ \\
\hline & & & ST199 (19A, 15B) + ST649 & $9.1(10)$ & 0 & 0 \\
\hline & & & ST425 (19F) & $3.6(4)$ & 0 & 0 \\
\hline & & & ST655 & $1.8(2)$ & 0 & $50(1)$ \\
\hline & & & ST177 (19F) + ST646 & $1.8(2)$ & 0 & $50(1)$ \\
\hline & & & ST644 & $0.9(1)$ & $100(1)$ & $100(1)$ \\
\hline & & & ST43 (19F) & $0.9(1)$ & 0 & $100(1)$ \\
\hline & & & ST423 (19F) & $0.9(1)$ & 0 & 0 \\
\hline $22 \mathrm{~F}$ & $6(85.7)$ & 31 & ST433 (22F) & $97.3(36)$ & 0 & $8.3(3)$ \\
\hline $23 \mathrm{~F}$ & $64(100)$ & 0 & $\begin{array}{l}\text { ST33 (23F) + ST37 (23F) + ST625 + ST626 + ST627 } \\
\quad+\text { ST628 + ST629 }\end{array}$ & $7.9(46)$ & $54.3(25)$ & $63(29)$ \\
\hline & & & ST81 (23F, 19F, 19A, 9V, 14) & $17.2(11)$ & $100(11)$ & $100(11)$ \\
\hline & & & ST242 (23F, 19F) & $6.3(4)$ & $100(4)$ & $100(4)$ \\
\hline & & & ST661 & $1.6(1)$ & 0 & 0 \\
\hline $33 \mathrm{~F}$ & $7(100)$ & 0 & ST100 (33F) + ST636 & $100(7)$ & 0 & $50(3)$ \\
\hline $35 \mathrm{~B}$ & $2(100)$ & 14 & ST451 & $75(12)$ & $100(12)$ & $25(3)$ \\
\hline & & & ST 452 & $25(4)$ & 0 & 0 \\
\hline 38 & $5(100)$ & 0 & ST393 (38) & $80(4)$ & 0 & 0 \\
\hline NT & 0 & 2 & ST662 + ST668 & $50(1)$ & $50(1)$ & $50(1)$ \\
\hline Total & 855 & 313 & & $89.2(1,042)$ & & \\
\hline
\end{tabular}

${ }^{a}$ Based on BURST analysis.

${ }^{b}$ Based on a combination of MLST and PFGE data. The cumulative data indicate that the majority of isolates that are within the same serotype and that also have a dice coefficient similarity of $>80 \%$ are within the same CC or single ST lineage (five or more alleles in common with and other member[s] of the complex). 


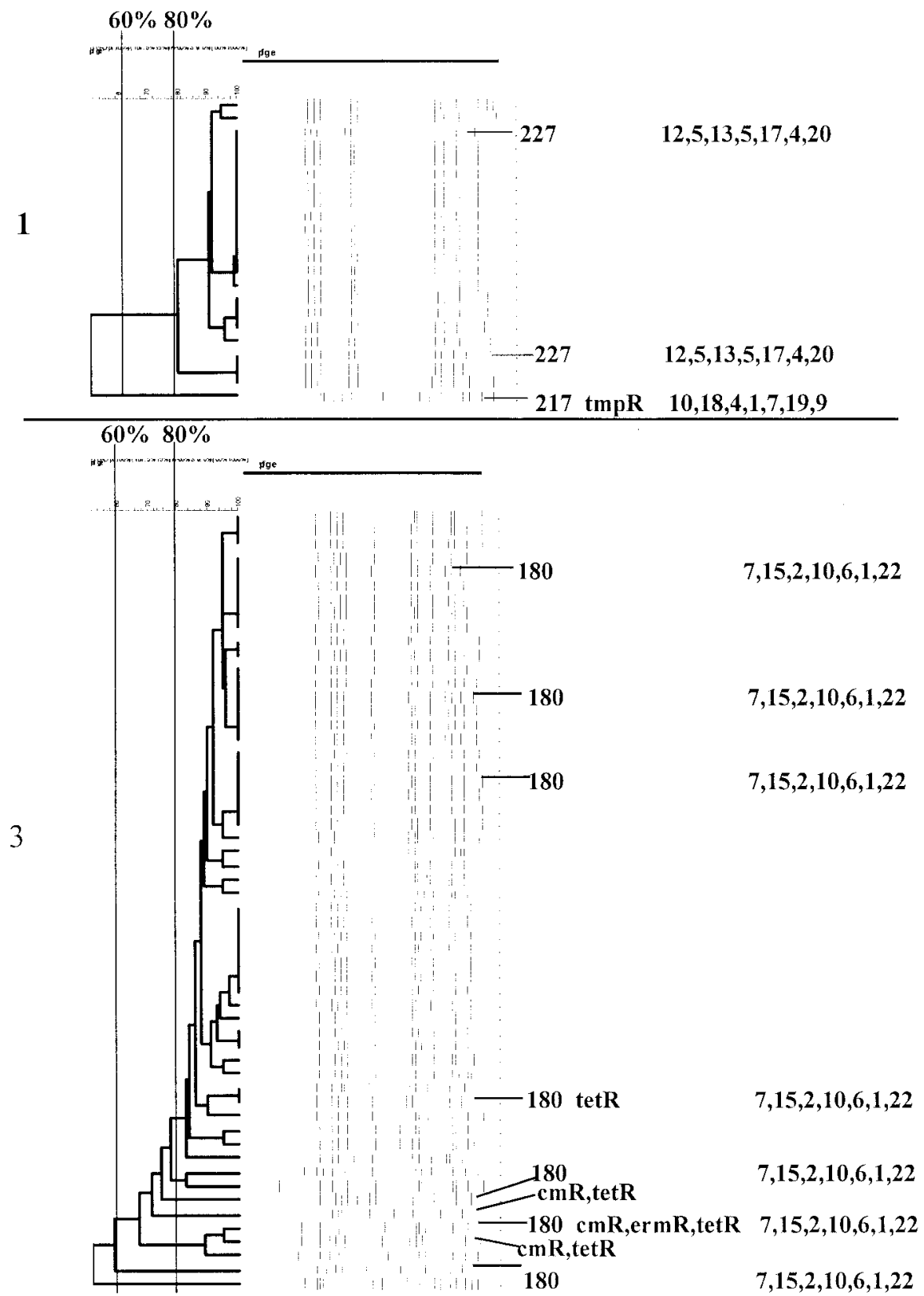

FIG. 1. PFGE dendrograms within serotypes included in this study accounting for multiple invasive isolates. Serotypes 1 and 3 are shown. The numbers of isolates represented in each dendrogram correspond to the total values for the corresponding serotypes given in Tables 3 and 4 . The serotype is indicated beside each dendrogram. All MLST results that have been determined are shown with the ST designation and antibiotic resistance profile (if any), followed by seven numbers specifying the alleles. Dice coefficient values (percentages) are indicated above each PFGE dendrogram. The vertical black lines extending various lengths of some of the dendrograms indicate penicillin-resistant (penR) isolates (MIC, $\geq 2.0$ $\mu \mathrm{g} / \mathrm{ml}$ ). Intermediate penicillin resistance (penI) is depicted by vertical gray lines. All antibiotic resistances among the isolates are indicated for serotypes that do not include penicillin-resistant isolates. Divergent type B $d d l$ alleles, found only among certain penicillin-resistant strains, are indicated by asterisks. cmR, chloramphenicol resistance; tmpR, trimethoprim-sulfamethoxazole resistance; ermR, erythromycin resistance; tetR, tetracycline resistance; cliR, clindamycin resistance; cefR, cefotaxime resistance; lfxR, levofloxine resistance.

isolates of differing serotypes, we subjected representative isolates to MLST to determine if they were genetically related.

Isolates that had five or more identical alleles almost always also had similar PFGE patterns with UPGMA dice coefficients of $\geq 80 \%$ (intraserotype relationships shown in Fig. 1 to 13 ; data not shown for isolates of differing serotypes). This relationship was tested as follows. In 19 of the dendrograms in- cluding 40 PFGE clusters (individual PFGE results differing by $\leq 20 \%$ similarity) of 2 or more isolates, 121 isolates were subjected to MLST to explore the relationship between the PFGE cluster and the allelic profile. In 37 of the 40 PFGE clusters, all isolates subjected to MLST had five to seven allelic identities. An exception was seen within a related PFGE cluster in serotype 6B, where ST641 and ST384 differed at four of seven 


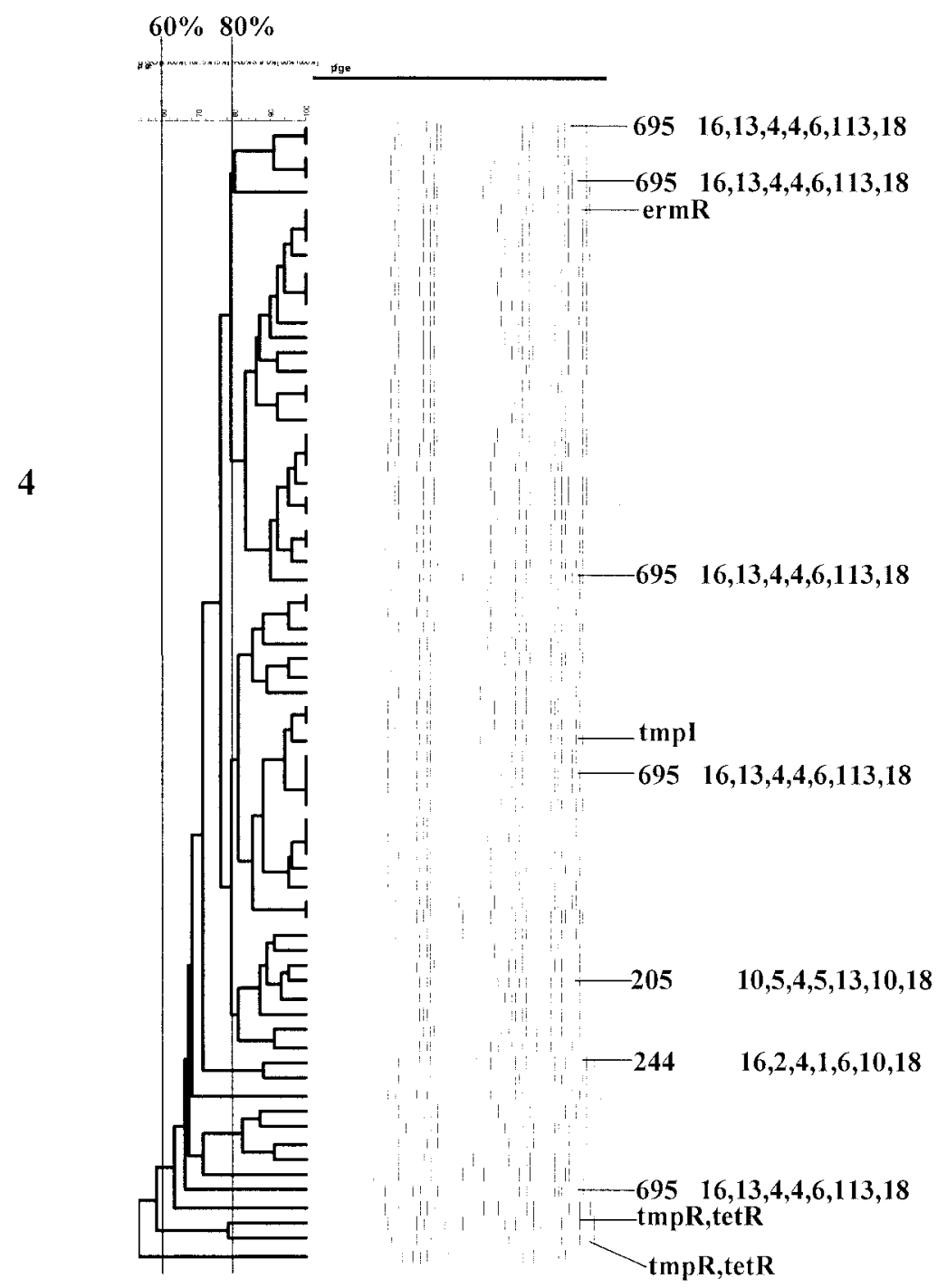

FIG. 2. Serotype 4. For details, see the legend to Fig. 1.

MLST loci (Fig. 4). Within serotype 4, ST695 and ST205 were both found within a related cluster and differed at five of seven MLST loci (however, note that the xpt-113 allele [GenBank accession no. AF474331] is a deletion derivative of xpt-10, found in ST205) (Fig. 2). In serotype 19A, ST172 and ST75 displayed three allelic differences (Fig. 10). Two other exceptions were seen. One large PFGE cluster within serotype 9V included ST156 (found twice), ST166 (found once), ST642 (found once), and ST643 (found twice) (Fig. 6). Although all four of these STs lie within BURST CC156 (Table 3; BURST analysis is explained in the next section), ST642 differed by four loci from ST156 and by three loci from ST166. The final exception was seen within a large serotype 18C cluster and concerned ST113 (found four times), ST637 (found once), ST638 (found twice), and ST648 (found once) (Fig. 9). Although these four STs lie within CC113 (Table 3), ST648 differs by three loci from both ST113 and ST637. In contrast, there were no exceptions to the general $\geq 80 \%$ PFGE relatedness; five to seven allelic identity relationship were seen within all 32 PFGE clusters tested within serotypes 1, 3, 6A, 7F, 9N,
11A, 12F, 14, 19F, 22F, 23F, 33F, 35B, and 38 and serogroup 15 (Fig. 1, 3, 5, 7, 8, 9, 11, 12, and 13).

Among the 121 MLST results that comprised multiple examples of testing within single PFGE clusters composed of profiles with $\geq 80 \%$ relatedness, only 12 STs representing seven pairs of ST comparisons (ST641-ST384, ST695-ST205, ST642-ST156, ST642-ST166, ST648-ST113, ST648-ST647, and ST172-ST75) did not exhibit five or more allelic identities (Fig. $2,4,6$, and 9). Note that only two of these seven pairs represented pairs of isolates that were not found within the same CC (ST641-ST384 and ST695-ST205) (Tables 3 and 4). Except for these two instances, all of the isolates tested within the same PFGE cluster were found to be within the same CC, even when they only had four or fewer allelic identities (Fig. 1 to 13; Tables 3 and 4). Typically representative of the PFGE clusterMLST relationship, for example, was the serotype 14 dendrogram, which revealed seven PFGE clusters of two or more isolates (Fig. 8). Within each cluster, the isolates tested (15 in total) invariably had five to seven allelic identities. Thus, selected isolates and reference strains within the same serotype 


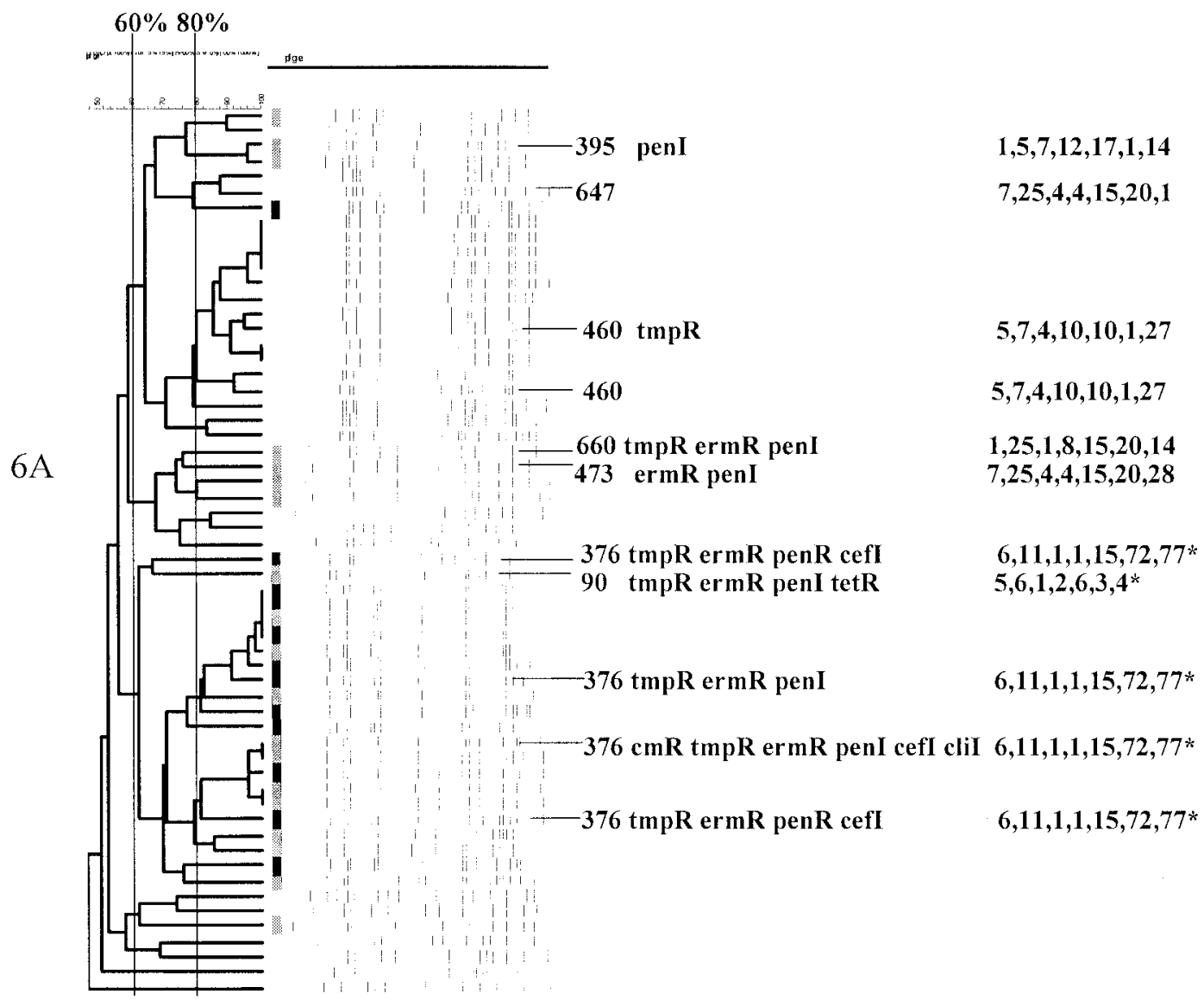

FIG. 3. Serotype 6A. For details, see the legend to Fig. 1.

exhibiting PFGE profiles with $\geq 80 \%$ similarity nearly always had five to seven allelic identities. On the basis of this observation, combined with a BURST analysis of 187 MLST results (105 different STs) representing the study isolates and reference strains, we were able to assign MLST-based CCs or lineages to $1,042(89.2 \%)$ of the 1,168 isolates subjected to PFGE (Tables 2, 3, and 4).

CCs and single ST lineages assigned through BURST analysis. In order to delineate sets of related STs, we found that BURST analysis (available at http://outbreak.ceid.ox.ac.uk /software.shtml) best served our purpose. BURST analysis is a simple yet powerful algorithm $(10,13)$ that is used to delineate CCs based on defined relationships between multilocus STs and is also used to predict ancestral STs within complexes. In this study, a CC simply consists of a set of isolates in which each isolate has five or more MLST target sequences in common with at least one other member of the set. BURST is based on the assumption that a successful ancestor strain becomes abundant and, through mutation and/or recombination, generates single-locus variants (SLVs). It follows that the ancestral ST is defined through its display of the greatest number of SLVs within the CC. Since there are more than 50 known sequence variant alleles for each of the seven targets (more than 100 for two of them), the likelihood of any pair of isolates having five alleles in common by chance appears remote $(<3 \times$ $10^{-8}$ if all were to occur at the same frequency).

Although the relatively limited numbers of STs (from the study isolates plus those of the reference strains in Table 1) used to construct Tables 3 and 4 seriously limited the robustness of BURST analysis for the purpose of determining ancestral STs, we felt that these data could only be presented clearly by limiting this algorithm as much as possible to the STs used in this study. When this was done, in several instances, the ancestral STs could not be assigned from the data set and instead assignments were predicted from BURST analysis of all known STs (CC81, CC156, CC218, CC124, CC473, CC62, and CC177 in Table 3). Despite this limited number of ST samples, reference STs predicted for CC199, CC236, CC662, and CC113 coincided with the ancestral ST generated by using all known allelic profiles (667 STs at the time of this study). Reference STs for CC146 and CC663 were chosen because of an unsurpassed number of SLVs or double-locus variants (DLVs) within their respective complexes; however, these STs were not actually identifiable as ancestral within a BURST analysis expanded to all known strains. Finally, a single CC (CC66) depicted an ancestral ST (based on a BURST analysis of all known STs) that was not actually encountered in this study (ST66), since the three STs in this study were DLVs of each other.

By using the cumulative PFGE and MLST data, we assigned CCs or lineages consisting of single STs to 67 to $100 \%$ of the isolates within each individual serotype (Table 2). For each serotype representing multiple isolates, 2 to 20 isolates were subjected to MLST. Single isolates that were sole representa- 


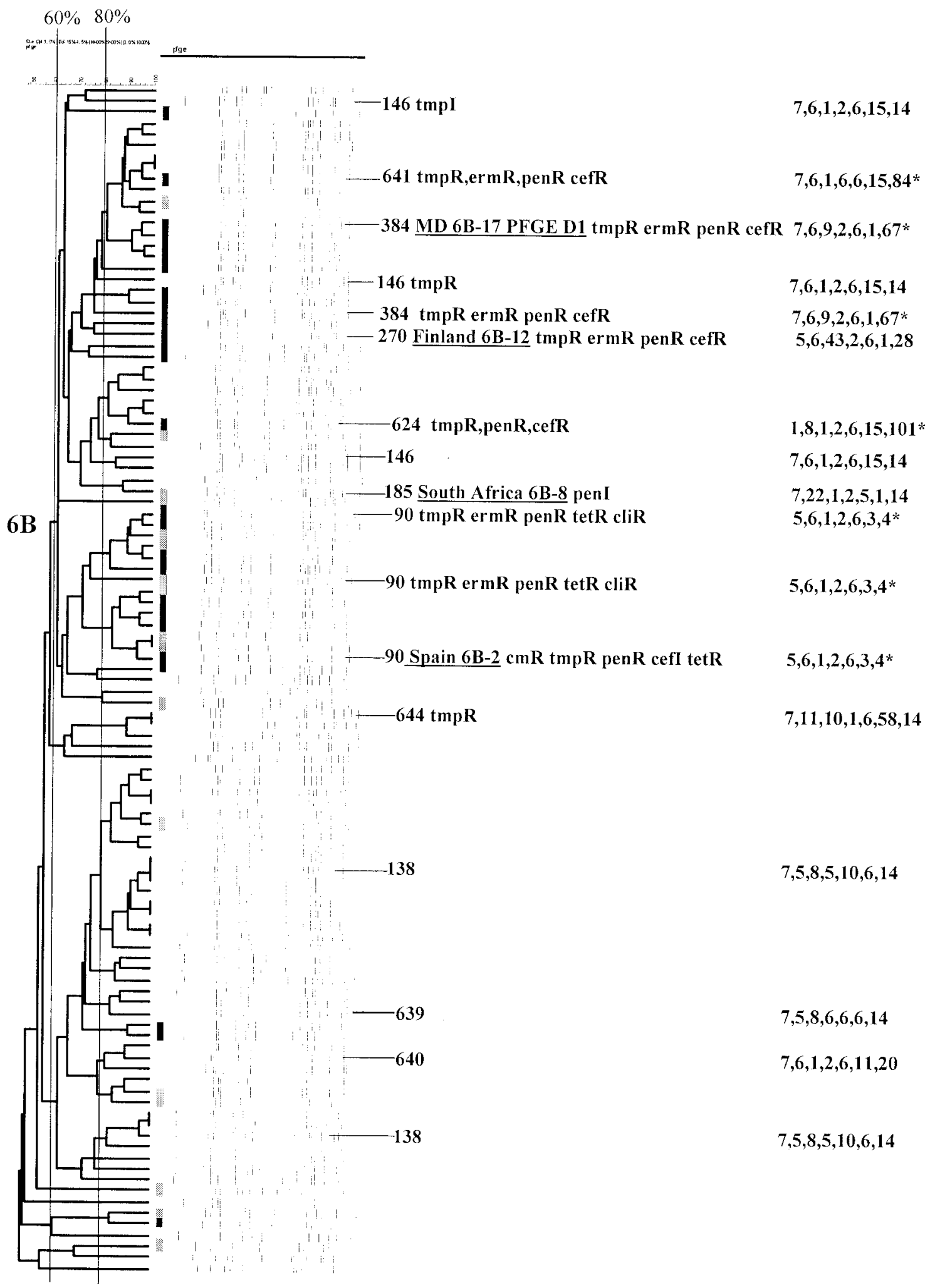

FIG. 4. Serotype 6B. Reference strains representing ST384, ST270, ST185, and ST90 are underlined. cefR, cefotaxime resistance. For details, see the legend to Fig. 1. 

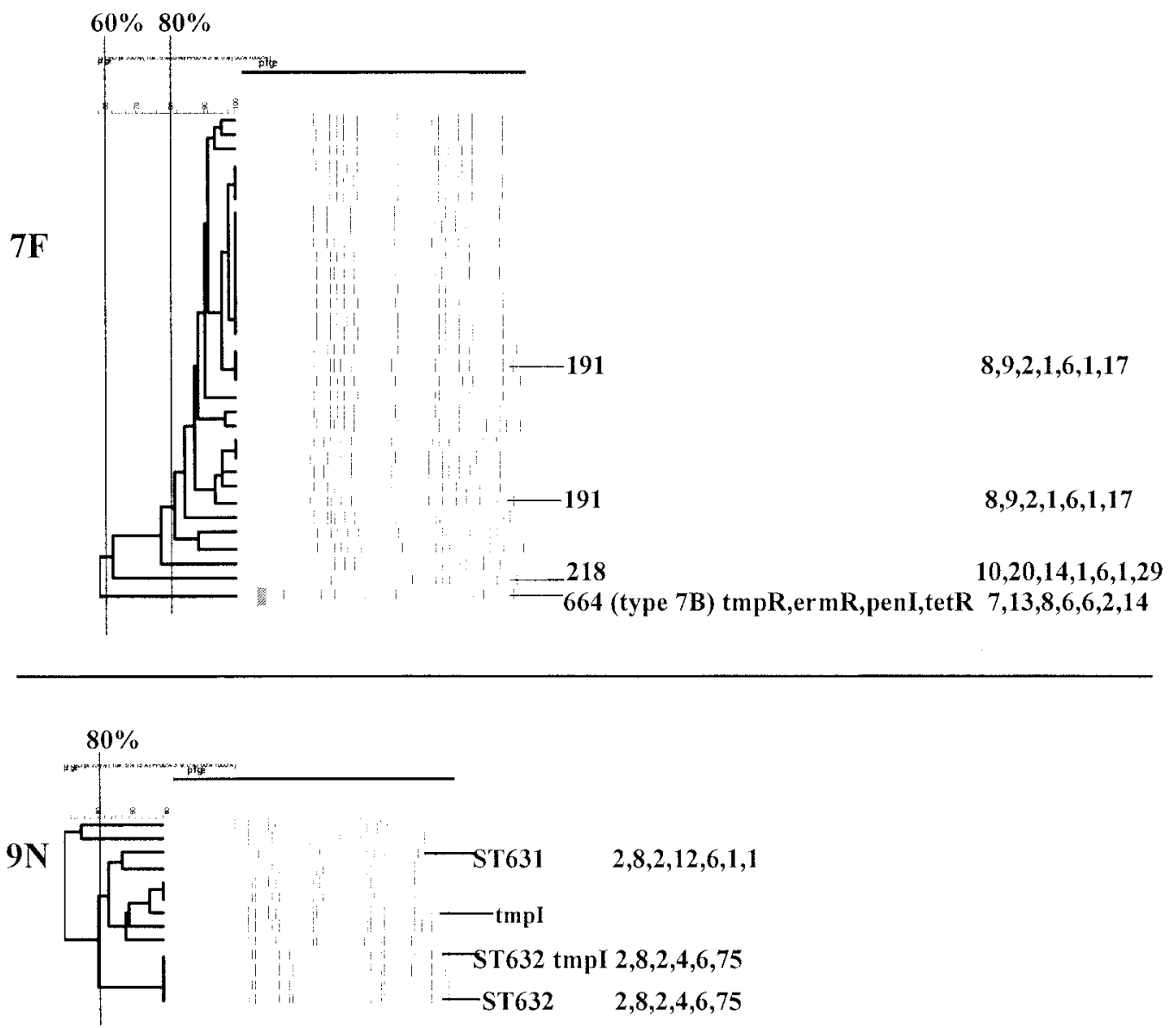

FIG. 5. Serotypes 7F and 9N. For details, see the legend to Fig. 1.

tives of serotypes (types 5 and 7B) were also subjected to MLST. A total of 50 CCs and single ST lineages were assigned on the basis of a BURST analysis of the 105 STs from the study isolates and reference strains (Tables 3 and 4). Seventeen CCs were represented by 2 to 10 related allelic profiles, while 33 lineages consisted of single STs with four or fewer allelic identities to other STs found in the sample set of study isolates and reference strains.

Upon performance of BURST analysis on all known allelic profiles (49 new STs plus the 618 STs recorded at www.mlst.net as of 27 November 2002), the number of single allelic profiles depicted as unique lineages in Table 4 was reduced from 33 to 8 (ST376, ST452, ST660, ST665, ST659, ST41, ST175, and ST448 [the latter 3 were associated with reference strains described in Table 1 only]). In addition, the number of CCs consisting of two or more STs was decreased from 17 to 13 when all known STs were included in the BURST analysis. In this manner, CC13, CC663, ST18, ST43, ST173, and ST226 (together constituting $20 \mathrm{STs}$ ) were merged into one large complex that included 88 additional STs in the MLST database (data not shown). Similarly, CC37, CC146, CC124, ST90, ST185, and ST384 (together constituting 13 STs) were merged together into one large complex that also included 88 additional STs). Finally, CC81 and CC66 (together constituting 7 STs) were merged into one complex that included 23 additional STs from the MLST database. Since such global rela- tionships based on BURST analysis would be very difficult to depict graphically, and our primary interest was the description of this population-based set of invasive isolates within our surveillance, we present the BURST data based primarily on the 105 STs represented by our isolate set and reference strains (Tables 3 and 4). SLVs and DLVs of indicated ancestral or reference STs are depicted, as are relationships between other STs included within given CCs (solid lines and dotted lines, respectively). We do not depict an UPGMA-generated dendrogram of the MLST results because the method generated easily discernible artifacts (data not shown; see http://outbreak .ceid.ox.ac.uk/software.shtml for details of why this occurs).

Allelic profile associations with specific serotypes. With few exceptions, serotype-ST associations were remarkably consistent between our data set and the extensive database available at www.mlst.net, indicating strong global associations of given STs with specific serotypes. We did find several additional serotype associations within individual STs; however, in the majority of cases, these results were still consistent with the observed serotype associations within the respective CCs. For example, within ST199, serotype 15C had not been previously reported at the database; however, serotypes $15 \mathrm{~B}$ and $15 \mathrm{C}$ are known to interconvert (33) and the association of type $15 \mathrm{C}$ isolates with ST199 has recently been reported (24). Although we did not see serotype 19F ST9 and ST485 isolates, there are two other STs within CC13 that were found in type 19F isolates 


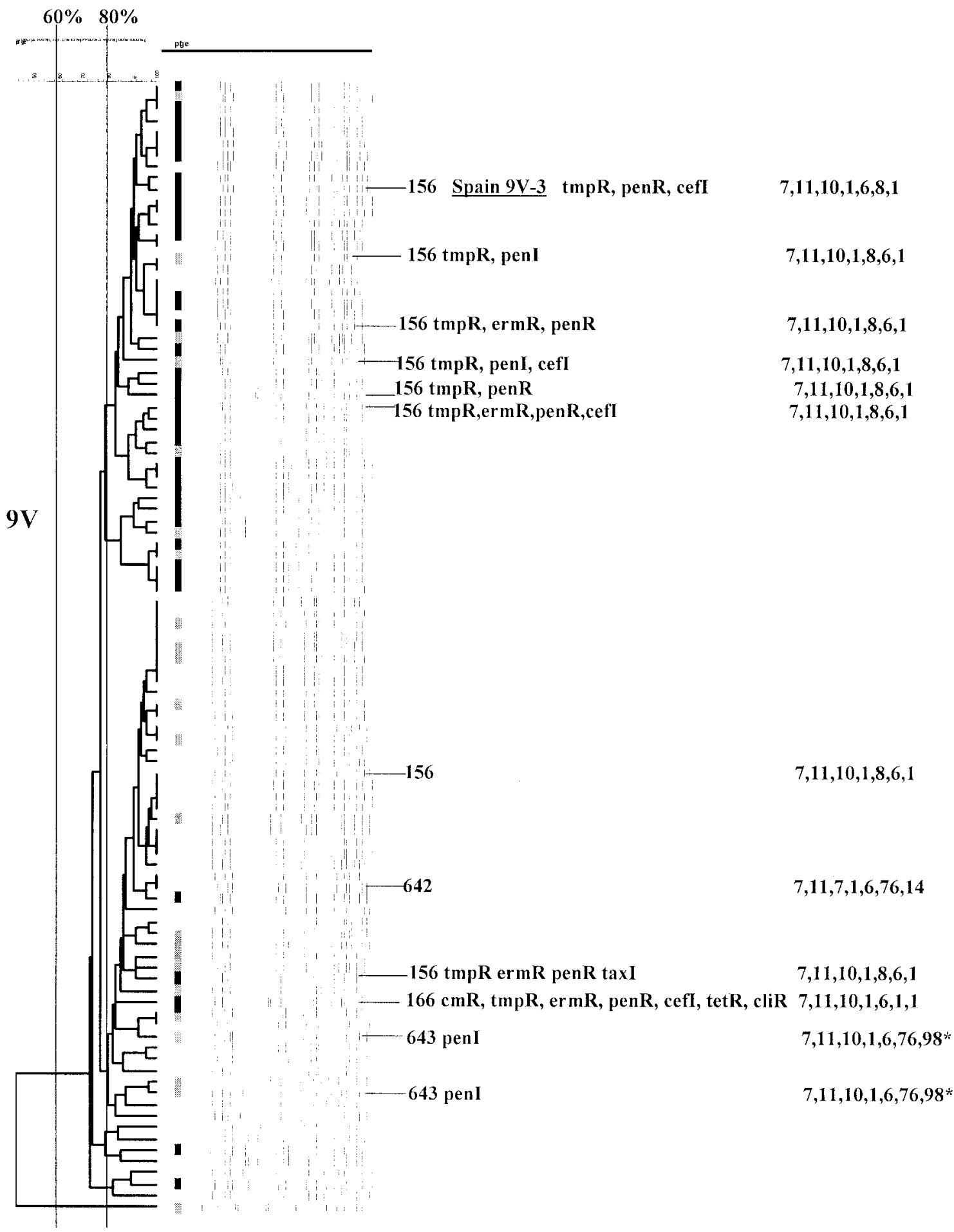

FIG. 6. Serotype 9V. The reference strain representing ST156 is underlined. For details, see the legend to Fig. 1.

(Table 3). Within CC218, we observed a novel serotype 7F isolate with ST218, which was formerly only associated with serotype $12 \mathrm{~F}$ (Table 3 ). Within lineages consisting of single STs (defined by the presence of four or fewer identities with other study isolates and reference strains), we found only one example of our results differing in serotype associations from those documented at the MLST database (Table 4). ST90, formerly found only within type $6 \mathrm{~B}$ isolates, was found in this study among type $6 \mathrm{~A}$ and $6 \mathrm{~B}$ isolates.

New MLST allelic profiles and alleles. During this work, we discovered 28 new MLST alleles not present at www.mlst.net and deposited them in that database. These included the alleles $d d l-75$ to $d d l-78, d d l-82$ to $d d l-84, d d l-95, d d l-98, d d l-99$, $d d l-101, x p t-72, x p t-75$ to $x p t-78, x p t-85, x p t-86, x p t-96, x p t-113$, 

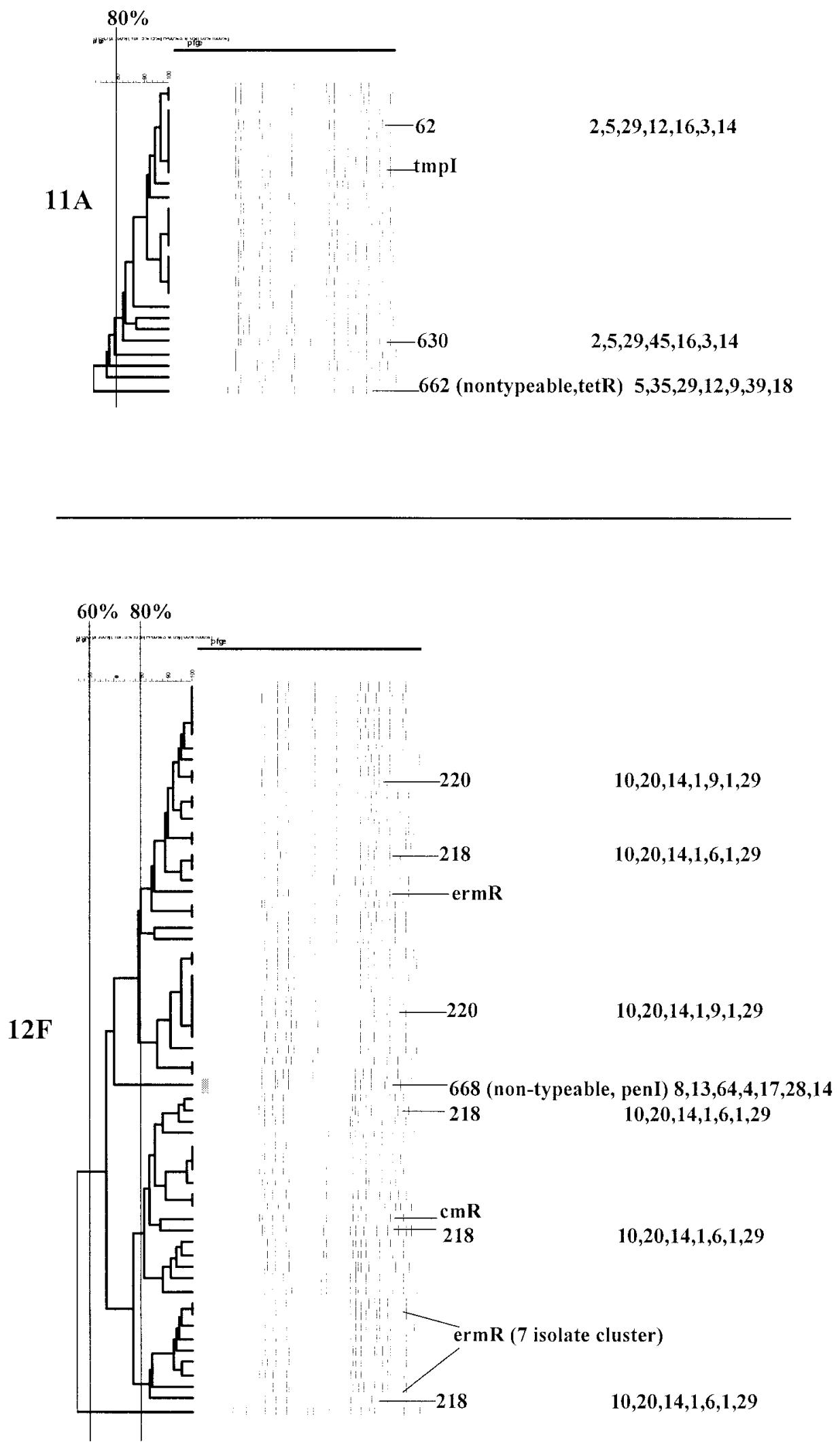

FIG. 7. Serotypes $11 \mathrm{~A}$ and $12 \mathrm{~F}$. For details, see the legend to Fig. 1. 


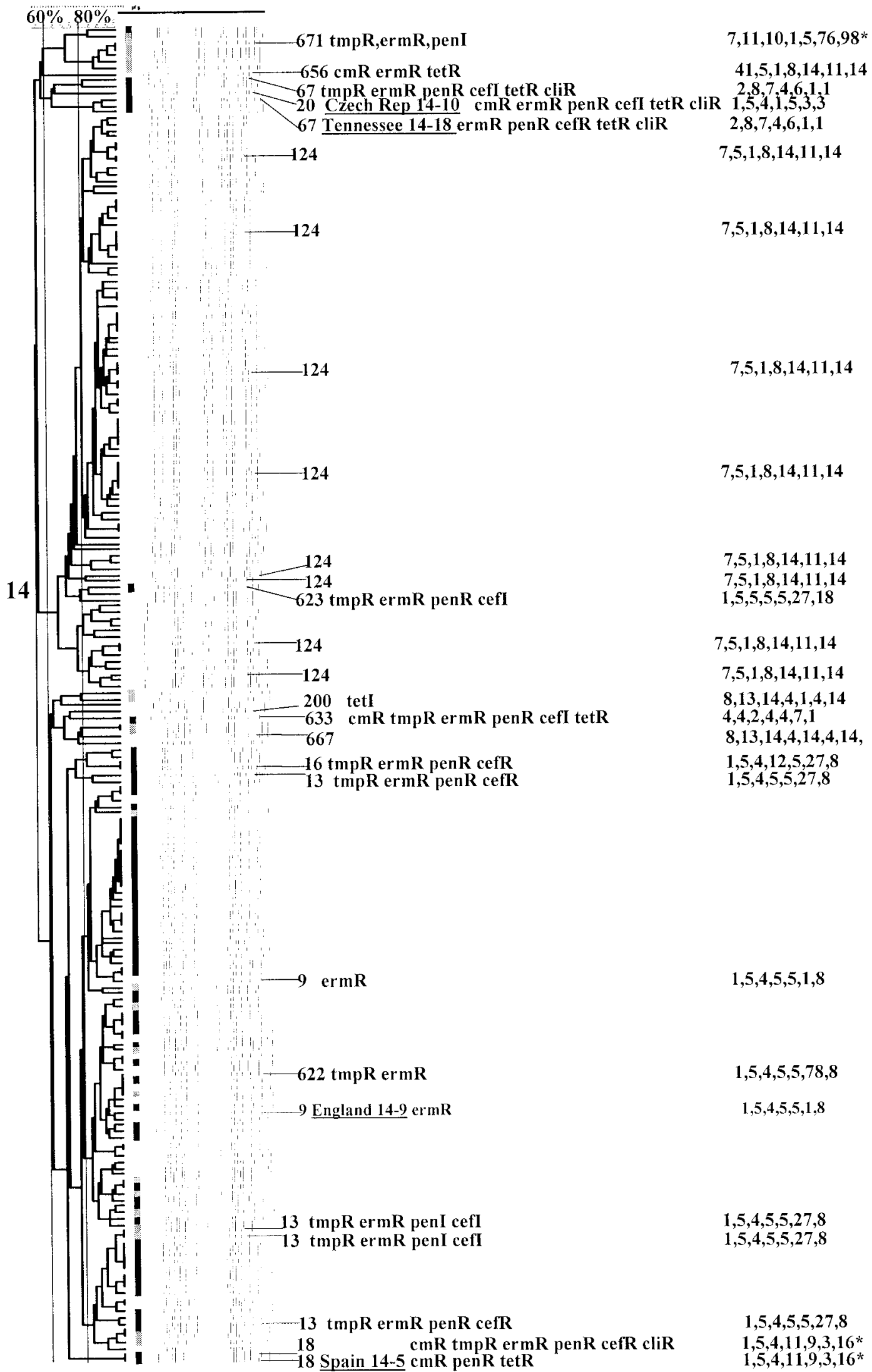

FIG. 8. Serotype 14. Reference strains representing to ST20, ST67, ST9, and ST14 are underlined. cliR, clindamycin resistance. For details, see the legend to Fig. 1. 

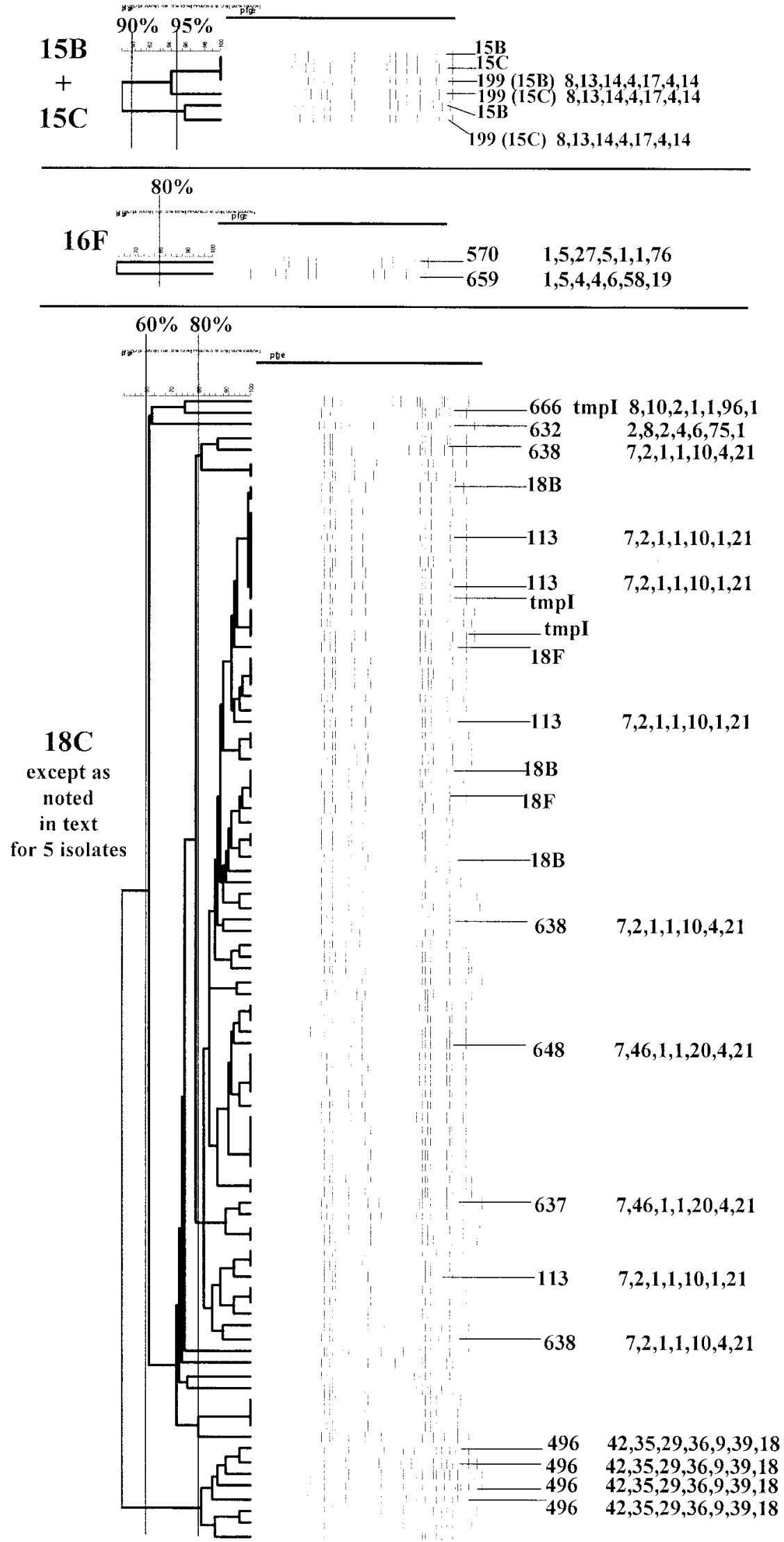

FIG. 9. Serogroup 15, serotype 16F, and serogroup 18. For details, see the legend to Fig. 1. 


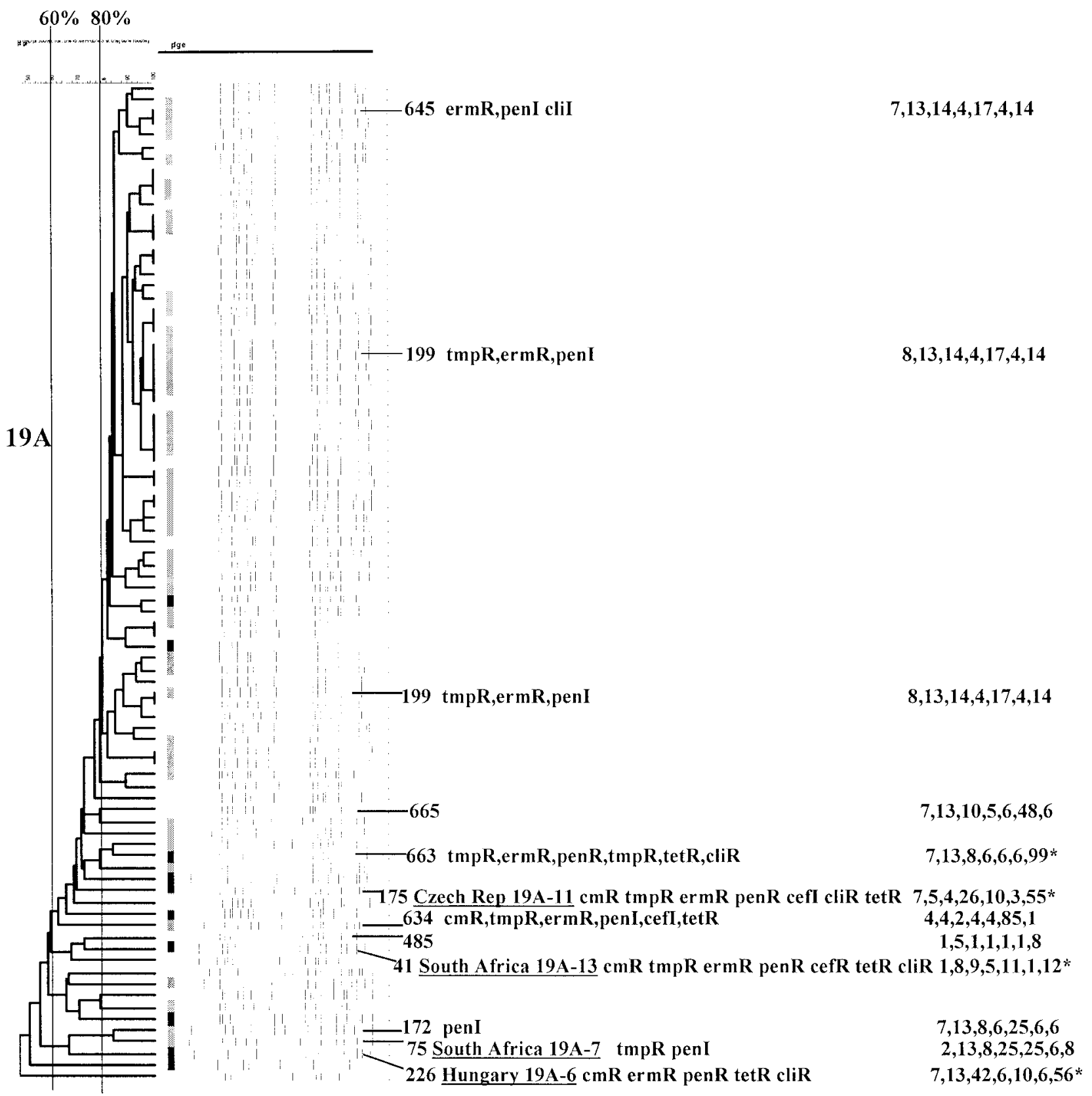

FIG. 10. Serotype 19A. Reference strains representing ST175, ST41, ST75, and ST226 are underlined. cliR, clindamycin resistance. For details, see the legend to Fig. 1.

recP44, recP45, aroE41, aroE42, gdh-46, gki-64, and spi-55. Twelve of these new alleles were independently corroborated by others, since they subsequently appeared in STs entered at www.mlst.net after we deposited them.

Seven of the new $d d l$ alleles from $\beta$-lactam-nonsusceptible isolates were relatively highly divergent (indicated by asterisks in Fig. 3 [serotype 6A], 4 [serotype 6B], 6 [serotype 9V], 8 [serotype 14], 10 [serotype 19A], 11 [serotype 19F], 12 [serotype 23F], and 13 [serotype $35 \mathrm{~B}$ ]) from each other and from pre-antibiotic era type A pneumococcal $d d l$ alleles. Type B alleles have about 90 to $97 \%$ sequence identity to type A alleles, while type A alleles generally have $>99 \%$ sequence identity to each other.
Our results are in agreement with previous work documenting the presence of divergent $d d l$ sequences in a subset of $\beta$-lactam-resistant isolates because of cotransformation with $p b p 2 b$ from nonpneumococcal donors (9). We did not encounter type $\mathrm{B} d d l$ alleles in any of the $\beta$-lactam-sensitive isolates subjected to MLST, which is also consistent with the "hitchhiking" effect of type B $d d l$ alleles linked with divergent $p b p 2 b$ sequences (9).

We present 49 new STs, 47 of which were assigned numbers ranging between 622 and 695 and 2 of which are the recently described STs ST451 and ST452 (1), which were originally discovered during this survey of year 1999 ABCs isolates (Fig. 13). In 11 additional instances, unique STs were corroborated 


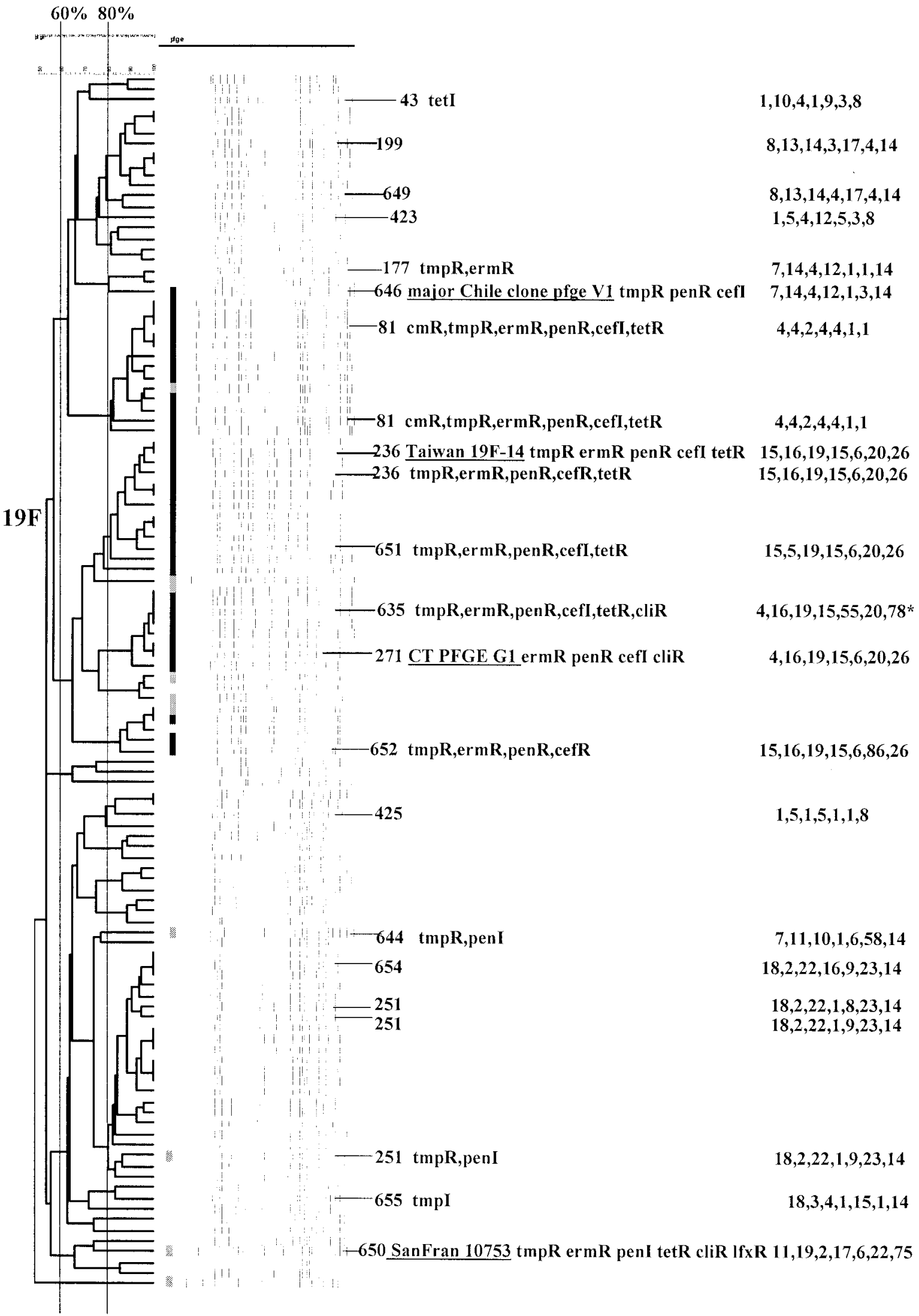

FIG. 11. Serotype 19F. Reference strains representing ST646, ST236, ST271, and ST650 are underlined. lfxR, levofloxacin resistance. For details, see the legend to Fig. 1. 

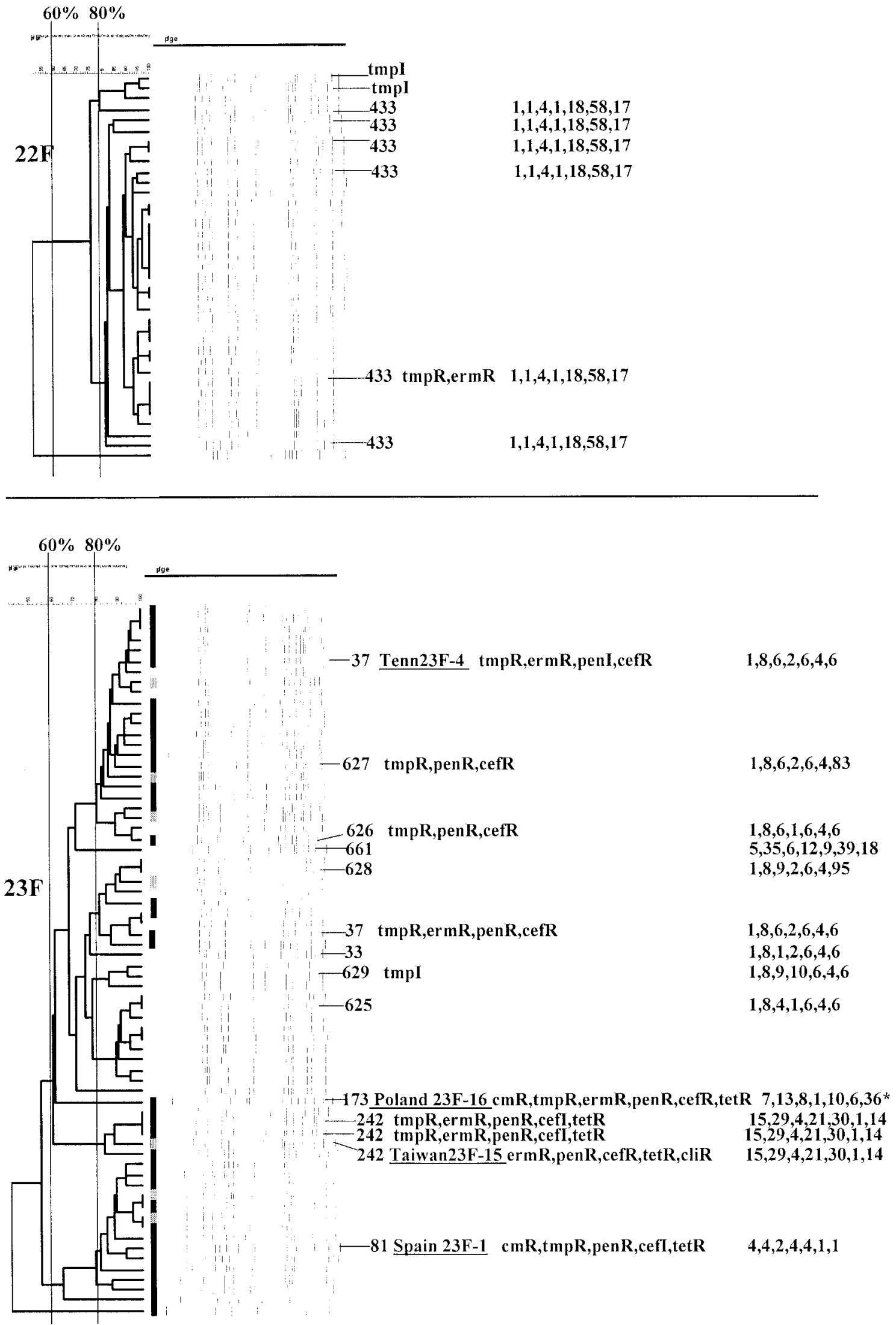

FIG. 12. Serotypes $22 \mathrm{~F}$ and $23 \mathrm{~F}$. Serotype $23 \mathrm{~F}$ reference strains corresponding to ST37, ST173, ST242, and ST81 are underlined. cliR, clindamycin resistance. For details, see the legend to Fig. 1. 

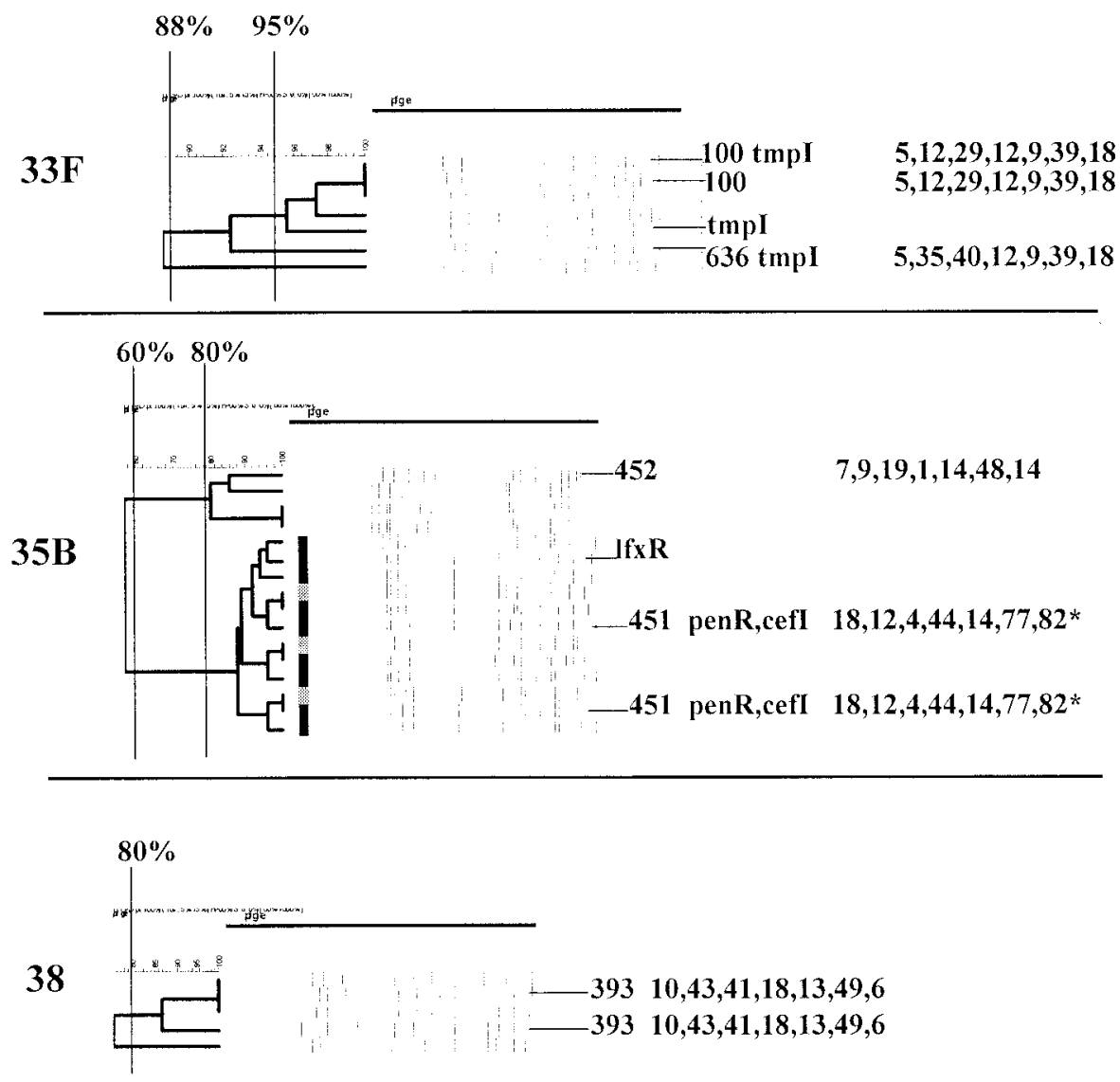

FIG. 13. Serotypes 33F, 35B, and 38. For details, see the legend to Fig. 1.

by independent researchers, since we encountered these profiles (three of which contained new alleles that we supplied to the database) prior to their becoming publicly available at www .mlst.net (ST376 [xpt-72], ST393, ST395, ST423, ST433, ST460, ST473, ST485, ST496 [aroE42], ST448, and ST570 [ddl-76]). With 10 of these $11 \mathrm{STs}$, the ST-serotype associations that we observed were in agreement with the independently listed documentation at www.mlst.net for these STs. The single exception was an invasive ST485 isolate of type 19A, while the carriage isolate described at www.mlst.net was type 19F.

Antibiotic-resistant isolates. Penicillin-resistant (MIC, $\geq 0.12$ $\mu \mathrm{g} / \mathrm{ml}$ ) isolates were restricted among serogroups $6,9,14,19$, and 23 , with the exception of serotypes $35 \mathrm{~B}$ (12 isolates) and 7B (1 isolate) (Fig. 5 and 13 and Table 2). An additional nontypeable isolate (ST668, described below within the description of serotype $12 \mathrm{~F}$ results) displayed intermediate penicillin resistance (Fig. 7). The majority of penicillin-resistant isolates (depicted by vertical broad lines in Fig. 3 to 5, 6 to 8, and 10 to 13) were resistant to various combinations of other antibiotics (depicted only for isolates subjected to MLST). For this reason, resistance to additional antibiotics is only indicated in Fig. 1 to 13 for serotypes in which resistance to any antibiotics occurs infrequently.

$\mathrm{CC}$ or lineage distribution within individual serotypes. (i) Serotype 1. Two lineages were apparent within serotype 1, each represented by a single ST (ST217 and ST227). Six of the seven year 1999 invasive isolates were available from children $\leq 5$ years of age in our 1999 invasive disease surveillance. In addition, we included 15 serotype 1 isolates from individuals ranging from 7 to 63 years of age. All serotype 1 isolates, except one trimethoprim-sulfamethoxazole-resistant isolate (Fig. 1), were sensitive to the eight antibiotics included in this analysis. Two isolates from within the major PFGE cluster of 20 isolates, and the single PFGE variant, were subjected to MLST, which revealed two nonrelated STs previously documented at www.mlst .net as being from serotype 1 invasive isolates in other countries (ST217 from Denmark and Sweden and ST227 from the United Kingdom and Denmark) (Fig. 1). The 20 isolates within the PFGE cluster that included 2 ST227 isolates were recovered in eight different states, while the sole unrelated ST217 variant was recovered from a child in Minnesota. Typical serotype 3 PFGE profiles highly associated with allelic profile ST180 had fortuitous similarity (UPGMA dice coefficients of up to $95 \%$ ) to the single serotype 1 PFGE profile that corresponded to ST217; however, MLST analysis indicated no relatedness (compare ST217 to ST180 from the serotype 1 and 3 dendrograms shown in Fig. 1). Thus, we found no evidence of genetic relatedness between serotype 1 isolates and isolates of other serotypes.

(ii) Serotype 3. From the PFGE and MLST data compiled from the 56 serotype 3 isolates from CDC surveillance (6 from children $<5$ years old and 50 from individuals $\geq 5$ years old representing all eight survey states), it is apparent that at least the majority of serotype 3 invasive isolates within the United 
TABLE 3. BURST analysis of reference STs combined with STs obtained from year 1999 invasive study isolates

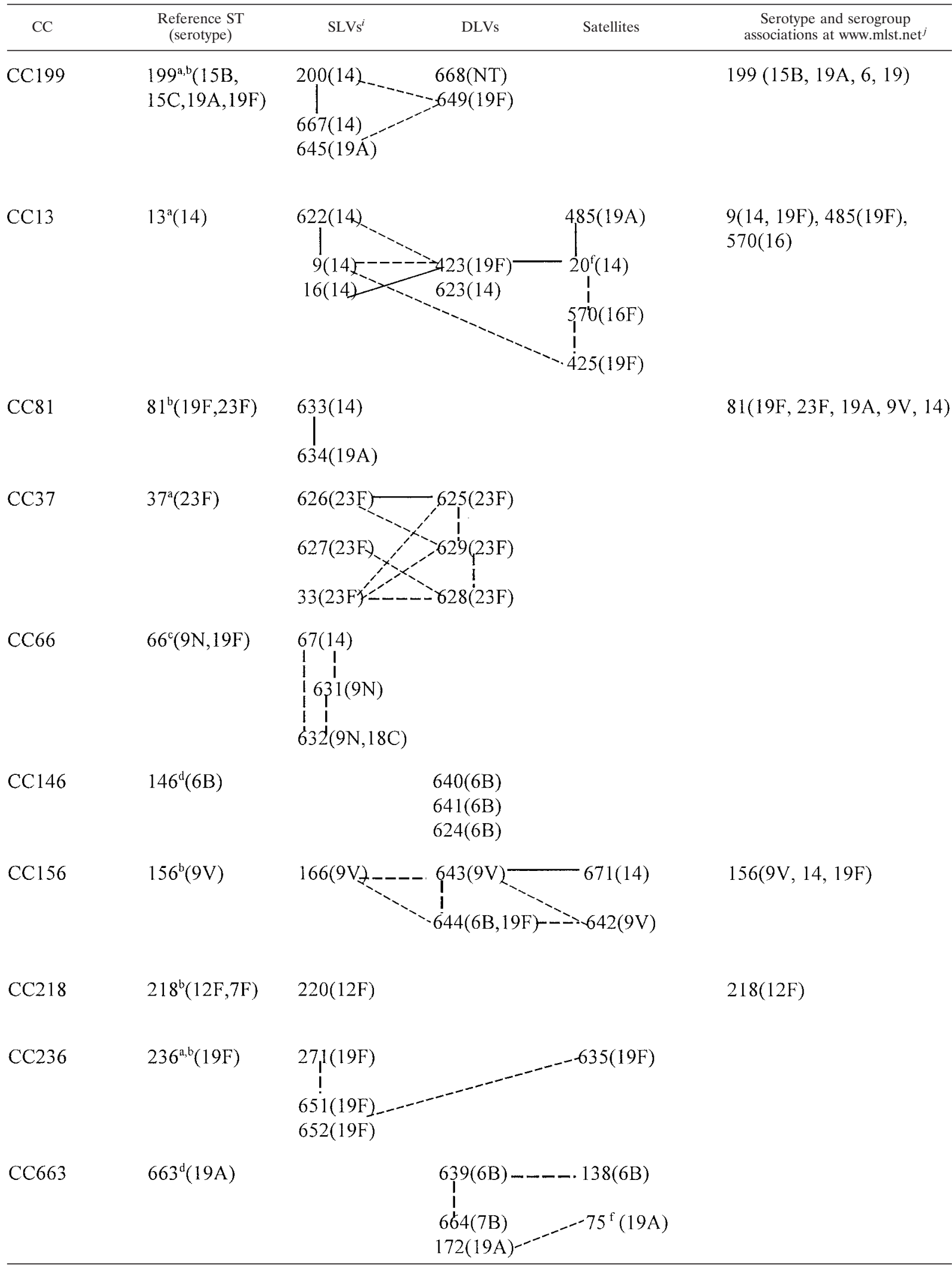


TABLE 3-Continued

\begin{tabular}{|c|c|c|c|c|c|}
\hline $\mathrm{CC}$ & $\begin{array}{c}\text { Reference ST } \\
\text { (serotype) }\end{array}$ & $\mathrm{SLVs}^{i}$ & DLVs & Satellites & $\begin{array}{l}\text { Serotype and serogroup } \\
\text { associations at www.mlst.net } t^{j}\end{array}$ \\
\hline CC662 & $\begin{array}{l}662^{a, b} \\
\text { (nontypeable) }\end{array}$ & $\begin{array}{c}100(33 \mathrm{~F}) \\
636(33 \mathrm{~F}) \\
\vdots \\
661(23 \mathrm{~F})\end{array}$ & $496(18 C)$ & & \\
\hline $\mathrm{CC} 251$ & $251^{\mathrm{e}}(19 \mathrm{~F})$ & $654(19 F)$ & & & \\
\hline $\mathrm{CC} 113$ & $113^{a, b}(18 C)$ & $\begin{array}{l}637(18 C) \\
638(18 C)\end{array}$ & & $648(18 C)$ & $113(18 \mathrm{~B}, 18 \mathrm{C})$ \\
\hline $\mathrm{CC} 124$ & $124^{b}(14)$ & $656(14)$ & & & \\
\hline $\mathrm{CC} 473$ & $473^{b}(6 A)$ & $647(6 \mathrm{~A})$ & & & \\
\hline CC62 & $62^{b}(11 A)$ & $630(11 \mathrm{~A})$ & & & \\
\hline $\mathrm{CC} 177$ & $177^{\mathrm{b}}(19 \mathrm{~F})$ & $646(19 F)$ & & & $177(19 F, 19)$ \\
\hline
\end{tabular}

${ }^{a}$ Ancestor ST based on BURST analysis of STs found in study isolates and reference strains.

${ }^{b}$ Ancestor ST found in study isolates for the expanded lineage when the BURST analysis was expanded to all known STs.

${ }^{c}$ Ancestor ST for the expanded lineage when the BURST analysis was expanded to all known STs; however, the ST was not encountered in study or reference isolates.

${ }^{d}$ This ST was chosen as a reference sequence because of the unsurpassed number of SLVs or DLVs within the CC but is not ancestral based on BURST analysis. The ancestor ST was not identifiable within a BURST analysis expanded to study isolates and reference strains. When the BURST analysis was expanded to all known STs, this merged two study CCs into one.

${ }^{e}$ When the BURST analysis was expanded to all known STs, these two STs were still the only members of the CC.

${ }^{f}$ Reference strain only.

${ }^{g}$ BURST analysis restricted to study and reference isolates only.

${ }^{h}$ Still only member of lineage upon BURST analysis of all known STs.

${ }^{i}$ Solid lines depict SLVs, and dotted lines depict DLVs.

${ }^{j}$ Only listed for those with associations different from those depicted in columns two to four from our results.

States are represented by a single clone with ST180 (Fig. 1 and Table 2), which has been documented in the global MLST database as being from type 3 isolates in at least six different countries. Four of the isolates depicted exhibited resistance to combinations of tetracycline, erythromycin, and chloramphenicol (Fig. 1).

(iii) Serotype 4. Approximately $85 \%$ (61 of 71 pediatric isolates) of type 4 isolates were projected to represent three distinct lineages represented by single STs (ST205, ST244, and ST695), each with four or fewer identical alleles in common with other STs seen in this study. BURST analysis using all known STs clustered ST244 and ST695 within the same lineage. ST695 (three-locus variant of ST244) was characterized by the novel xpt-113 allele with an in-frame deletion of 39 codons. ST695 was obtained from five independent isolates and represented approximately $72 \%$ of the type 4 isolates (three PFGE clusters and an additional isolate with a divergent PFGE profile) (Fig. 2 and Table 2). With the exception of the 39-codon deletion, $x p t-113$ is identical to $x p t-10$, which is common to both ST244 (two isolates) and ST205 (eight isolates). ST695 is an SLV (xpt-10 to xpt-113) of ST246 recorded at www.mlst.net as being from type 4 invasive isolates recovered in the United Kingdom during 1997.

One ST695 isolate was erythromycin resistant, and another isolate within the ST205 PFGE cluster was trimethoprim-sulfamethoxazole resistant. Two additional isolates of undeter- mined genetic types were trimethoprim-sulfamethoxazole resistant and tetracycline resistant.

(iv) Serotype 5. A single trimethoprim-sulfamethoxazoleresistant serotype 5 pediatric isolate was ST653, a DLV of a type 5 isolate recovered in Colombia in 1994 (www.mlst.net).

(v) Serotype 6A. Fifty-three percent (31 isolates) of the 58 serotype $6 \mathrm{~A}$ isolates were penicillin resistant. Fifty-seven of the 58 isolates were recovered from pediatric patients. The sole isolate from an adult patient was the result of an isolate mistakenly initially serotyped as $9 \mathrm{~N}$. This was a genetically heterogeneous isolate set represented by at least six distinct genetic lineages deduced from 11 MLST results (Fig. 3 and Tables 3 and 4). We discovered four of these STs independently (ST376, ST460, ST473, and ST395) prior to their appearance at www .mlst.net, which documented serotype $6 \mathrm{~A}$ isolates recovered between 1994 and 1998 (ST376 [from a case of meningitis; blood isolate], ST460 [unspecified diagnosis; sputum isolate], ST473, and ST395 [both carriage isolates]). ST376 and ST460 putatively accounted for more than $50 \%$ of serotype $6 \mathrm{~A}$ isolates (Fig. 3 and Table 2). ST376, circumstantially representing 19 isolates (Fig. 3), was characterized by the new type B $d d l-77$ allele and was obtained from 4 independent isolates. ST460 was obtained from 2 isolates representing a PFGE cluster of 13 penicillin-sensitive isolates. CC473 isolates (ST473 and its SLV ST647) represented five penicillin-resistant isolates. ST395 represented two penicillin-resistant isolates. ST660 and ST90, 
TABLE 4. STs differing by three or more alleles from all other STs found among year 1999 study isolates and reference strains ${ }^{a}$

\begin{tabular}{l} 
ST \\
$\begin{array}{c}\text { Serotype and serogroup } \\
\text { associations at www.mlst.net }\end{array}$ \\
\hline ST18 (14) \\
ST41 ${ }^{c, d}(19 \mathrm{~A})$ \\
ST43 (19F) \\
ST90 (6A, 6B) \\
ST173 ${ }^{c}(23 \mathrm{~F})$ \\
ST175 ${ }^{c, d}(19 \mathrm{~A})$ \\
ST180 (3) \\
ST185 (6B) \\
ST191 (7F) \\
ST205 (4) \\
ST217 (1) \\
ST226 (19A) \\
ST227 (1) \\
ST242 (23F)
\end{tabular}

${ }^{a}$ BURST analysis restricted to study and reference isolates.

${ }^{b}$ Only those with associations that differed from our results are listed.

${ }^{c}$ Reference strain only.

${ }^{d}$ Still only member of lineage upon BURST analysis of all known STs.

${ }^{e} \mathrm{NT}$, nontypeable.

both with four or fewer similarities to all of the other STs in this study, were found in single isolates that had PFGE profiles dissimilar to other type 6A isolates. ST90 was additionally found among serotype $6 \mathrm{~B}$ isolates.

(vi) Serotype 6B. One hundred three serotype 6B isolates (all pediatric) were analyzed, constituting at least six CCs or single ST lineages (nine STs). Forty-two percent $(n=43)$ of these isolates were penicillin resistant. CC663 ST138 (documented as being from invasive serotype $6 \mathrm{~B}$ isolates from three different countries in the MLST database) and ST639 represented $24(23.3 \%)$ of these isolates, all but 1 of which were penicillin sensitive.

Isolates with PFGE patterns and/or STs similar or identical to those of reference strains Spain $^{6 \mathrm{~B}}-2$ (ST90; 13 isolates), $\mathrm{MD}^{6 \mathrm{~B}}-17$ (ST384; 19 isolates), and S. Africa ${ }^{6 \mathrm{~B}}-8$ (ST185; 1 isolate) were also identified (Fig. 4 and Table 2).

ST644 (CC156) was found within single serotype 6B and 19F isolates (Table 3).

(vii) Serotype 7B. Only one serotype 7B isolate was analyzed, which was mistakenly included in this study as a serotype $7 \mathrm{~F}$ isolate (bottom of Fig. 5 serotype $7 \mathrm{~F}$ dendrogram). This isolate was intermediately penicillin resistant and was resistant to three additional antibiotics. The ST of this isolate (ST644) was found among CC663 isolates that include serotypes 19A and 6B (Table 3).

(viii) Serotype 7F. Thirty-one serotype 7F isolates were analyzed (seven from pediatric patients), all of which were antibiotic susceptible (Fig. 5). Twenty-nine (93.5\%) of these isolates putatively have STs identical or highly similar to ST191, which was previously found among invasive serotype $7 \mathrm{~F}$ isolates that have been recovered from multiple countries (see www.mlst.net). One serotype 7F isolate, with a PFGE profile markedly different from those of all other serotype $7 \mathrm{~F}$ isolates, was ST218 (within CC218), which was otherwise found only in serotype $12 \mathrm{~F}$ isolates (Table 3 ).

(ix) Serotype 9N. Only 2 of the 13 serotype $9 \mathrm{~N}$ isolates were from pediatric patients. These isolates and nine other isolates from adult patients made up the major PFGE cluster, which appeared to be composed of CC66 isolates (ST631 and ST632) (Fig. 5).

(x) Serotype 9V. Forty-two of the 98 serotype 9V isolates were initially mistakenly tested as belonging to other serogroup 9 serotypes (9N and 9A). In this manner, we fortuitously included 26 adult isolates in this analysis (Fig. 6). About $92 \%$ of these isolates (both pediatric and adult) were found to be within CC156 (Table 3, ST156, ST166, ST642, and ST643) and closely related to internationally disseminated clone Spain $^{9 \mathrm{~V}}-3$ (ST156). More than $63 \%$ of the CC156 isolates examined were penicillin resistant (Table 2). Two independent penicillin-resistant isolates were found to have in common ST644, a DLV of ST156 (Table 3) characterized by the previously undocumented $d d l-98$ and $x p t-76$ alleles. A single ST166 isolate was found. ST166, an SLV of ST156, was initially found in a multiresistant isolate recovered in Taiwan (see www.mlst.net). ST642 (which also carried new allele xpt-76) differed from the other STs found in CC156 isolates by two to four alleles.

(xi) Serotype 11A. Twenty-two of the 25 serotype 11A isolates $(88 \%$, all but one from adults) were putatively within CC62 (Table 3), which consists of ST62 (recorded at www.mlst.net as being from type 11A meningitis isolates recovered in Spain) and an SLV of ST62 characterized by the new recP45 allele (ST630) (Fig. 7). Antibiotic resistance within CC62 was restricted to one trimethoprim-sulfamethoxazole-resistant isolate. A single tetracycline-resistant isolate displaying a divergent PFGE profile was typed as ST662, which lies within CC662 (Table 3). This isolate, although originally typed as $11 \mathrm{~A}$, was subsequently found to be nontypeable. It is interesting that CC662 appears to consist primarily of type 18C and $33 \mathrm{~F}$ isolates (along with a single type $23 \mathrm{~F}$ isolate) and that, on the basis of BURST analysis, ST662 is the ancestral profile.

(xii) Serotype 12F. The 60 serotype $12 \mathrm{~F}$ isolates (11 from pediatric patients) were primarily within CC218 (Table 3), consisting of ST218 and ST220 (Fig. 7 and Table 2). Both of these profiles are associated solely with invasive type $12 \mathrm{~F}$ isolates at www.mlst.net (ST218 reported as being from several different countries and ST220 reported as being from a single isolate recovered in Denmark). One isolate initially typed as 12F had a divergent PFGE profile and divergent ST668, found within CC199 (Table 3). Subsequent reserotyping revealed this strain to be nontypeable (Fig. 7).

(xiii) Serotype 14. Serotype 14 is most often associated with invasive isolates recovered from children and consisted of 208 isolates recovered from pediatric patients $(63.5 \%$ of the year 
1999 type 14 invasive isolates recovered from children in our survey area).

On the basis of MLST results from seven serotype 14 isolates within a major related PFGE cluster (also including the ST9 reference strain England $\left.{ }^{14}-9\right)$, the single largest serotype 14 CC (96 isolates $=46.2 \%$ ) was $\mathrm{CC} 13$, in which $81.2 \%$ of the strains (78 isolates) were penicillin resistant (Fig. 8 and Tables 2 and 3). Besides ST9 (also found in penicillin-sensitive, internationally dispersed clone England $\left.{ }^{14}-9\right)$, these CC13 isolates were characterized by ST13 (characterized by both penicillinresistant and penicillin-sensitive isolates in the MLST database), ST16 (listed at www.mlst.net as being from a single pneumonia-causing serotype 14 isolate in Canada), ST622 (SLV of ST13 characterized by new allele xpt-78), and ST623 (DLV of ST13 associated with two isolates on the basis of PFGE) (Table 3).

The majority of penicillin-susceptible isolates (78 [71\%] of the 110 susceptible isolates) appeared to be within CC124, apparently primarily with ST124 in common (seven independent isolates had ST124). ST124 is documented in the MLST database as being from invasive type 14 isolates recovered from several different countries. An SLV of ST124 (ST656), characterized by the new aroE41 allele, was found within a single multiresistant isolate that displayed a divergent PFGE profile (Fig. 8, top of type 14 dendrogram).

Five multiresistant isolates were represented by ST671. This was the only ST representing a type 14 strain within the type 9V-dominated CC156 group (Table 3). This was not an unexpected result, since ST156 (the ancestor ST found in Spain $^{9 \mathrm{~V}}$-3) has also been documented among type 14 isolates $(7,25)$. Four multiresistant isolates putatively had in common ST67 (CC66 in Table 3) on the basis of two isolates with PFGE profiles highly similar to that of reference strain Tennessee $^{14}-10$ (ST67), and another pair of isolates had highly similar PFGE profiles (one of which was directly determined to be ST67) (Fig. 8).

CC199 serotype 14 isolates were composed of an ST200 tetracycline-resistant isolate with a unique PFGE profile and the SLV of ST200, ST667. ST200 has been previously documented in serotype 14 invasive isolates recovered in Denmark and Taiwan. One multiresistant isolate had a PFGE profile identical to that of reference strain Spain ${ }^{14}-5$ (ST18) and was directly typed as ST18, which has four or fewer allelic identities with other STs in the sample set (Table 4). A single multiresistant ST633 isolate (CC81) was also observed.

(xiv) Serotypes 15B and 15C. The six serogroup 15 isolates from pediatric patients that were available to us (from patients in Georgia, Minnesota, and Connecticut) had highly similar PFGE profiles and ST199 (the ancestral ST of CC199 in Table 3 on the basis of study STs and a cumulative BURST analysis of all known STs) (Fig. 9). ST199 was also found among serotype $19 \mathrm{~A}$ and $19 \mathrm{~F}$ study isolates and is documented at www .mlst.net as being from serotype $15 \mathrm{~B}$ and $19 \mathrm{~A}$ invasive isolates recovered in the United Kingdom and The Netherlands. As recently reported, ST199 is apparently commonly found within both serotype $15 \mathrm{~B}$ and $15 \mathrm{C}$ pneumococci recovered from nasopharyngeal specimens of children in the United Kingdom (24).

(xv) Serotype 16F. One of the two serotype $16 \mathrm{~F}$ isolates was from an adult and was originally mistakenly identified as sero- type 9A. Reserotyping this isolate revealed that it was serotype $16 \mathrm{~F}$. The ST of this isolate, which was characterized by our newly discovered $d d l-76$ allele, was independently corroborated on 21 October 2002 at www.mlst.net as being from a serogroup 16 isolate recovered in Scotland. The other serotype $16 \mathrm{~F}$ isolate (from a child) was the unique ST659 (Fig. 9). The closest known matches to ST659 have only three alleles in common and are from a variety of serotypes, none of which are type $16 \mathrm{~F}$.

(xvi) Serotype 18C. Ninety-three serotype $18 \mathrm{C}$ isolates from children were analyzed, five of which were mistakenly included and subsequently found to be serotype $18 \mathrm{~B}$ (three isolates) or $18 \mathrm{~F}$ (two isolates). These five isolates had PFGE profiles very similar to those of the majority of type $18 \mathrm{C}$ isolates. The major PFGE cluster, consisting of 74 isolates $(79.6 \%$ of the total) represents $\mathrm{CC} 113$ (Fig. 9, all 9 isolates tested). On the basis of a BURST analysis of the study STs and all known STs, ST113 is the ancestor ST within this CC, which also includes SLVs ST637 and ST638 (three independent isolates) and satellite ST648 (three-locus variant of ST113) (Table 3). This observation is consistent with ST113 being observed in four of the nine isolates within this PFGE cluster that were sequence typed. ST113 is documented as being from isolates dating back to 1980 that were recovered from meningitis isolates in the United Kingdom, The Netherlands, and Spain. ST496 (within CC662, which additionally represents type 33F, 11A, and 23F study isolates) was characterized by our new aroE42 allele and was found in four isolates within a total cluster of eight isolates that had highly similar PFGE profiles. This ST was independently entered at www.mlst.net on 10 July 2002 as being from a type 18C carriage isolate recovered in 1996 in Finland.

Single isolates with divergent PFGE profiles were also found. A unique trimethoprim-sulfamethoxazole-resistant ST666 isolate was characterized by the new xpt-96 allele and is a DLV of STs found in serotypes 14, 18F, and 21. An ST632 isolate (CC66 shared with type 14 and 9N isolates), characterized by the new xpt-75 allele, was additionally found in two serotype $9 \mathrm{~N}$ isolates in this study.

(xvii) Serotype 19A. Eighty-two serotype 19A isolates were analyzed, 18 of which were recovered from pediatric patients. For $54(65.8 \%)$ of these isolates, the penicillin MICs were $\geq 0.12 \mu \mathrm{g} / \mathrm{ml}$. On the basis of PFGE and MLST data shown in Fig. 10, 72\% $(n=59)$ of the type 19A isolates were within CC199 (see serogroup 15 above) and typed as ST199 (two independent isolates) and ST645 (an ST199 SLV) (Table 3). The lower $30 \%$ of the serotype 19A dendrogram shown in Fig. 10 represents a diverse set of reference strains and isolates representing at least three distinct CCs or single ST lineages (ST663, ST172, and ST75 representing CC663; ST665, which has four or fewer allelic identities to all known STs; and ST485 within CC13). ST172 and ST485 are listed at www.mlst.net as being from serotype 19A meningitis and serotype 19F carriage isolates, respectively.

(xviii) Serotype 19F. A set of 110 serotype 19F pediatric isolates were analyzed (Fig. 11) revealing a diverse population of at least 10 different CCs or single ST lineages (17 different STs from study isolates recorded in Table 2). Approximately $21 \%$ of the isolates were categorized as CC236 (23 multiresistant isolates), consisting of ST236 (ancestral sequence, associated with major resistant clone Taiwan ${ }^{19 F_{-}}$-14), ST271 (SLV of 
ST236 documented at www.mlst.net as being from invasive multiresistant strains in Korea and the United States), ST652 (SLV of ST236 with new allele xpt-86), ST651 (SLV of ST236), and ST635 (DLV of ST271 characterized by new allele spi-55 and new type B allele $d d l-78$ ) (Table 3).

Another $20 \%$ of serotype $19 \mathrm{~F}$ isolates (22 isolates, 21 of which were penicillin sensitive) were assigned to CC251, which consists of ST251 (found in three isolates, one of which was intermediately penicillin resistant) and ST654 (SLV of ST251 found in one isolate). ST251 is documented as being from an antibiotic-sensitive type 19F meningitis isolate recovered in the United Kingdom in 1995.

About 12\% (13 multiresistant isolates) of serotype 19F isolates were within CC81 since two isolates within this cluster had ST81 in common. ST81 is found within reference strain Spain $^{23 \mathrm{~F}}$-1 and was also presumed to be within multiple serotype 23F study isolates on the basis of PFGE profiles (Fig. 11).

Ten penicillin-sensitive isolates (9.1\% of serotype $19 \mathrm{~F}$ isolates) were within CC199 (ancestral ST199 and DLV ST649, Tables 2 and 3 ).

Multiple serotype 19F isolates were also represented within CC13 (ST425 [four isolates] and ST655 [two isolates]) and CC177 (two isolates; one isolate was typed as ST177 and, with another isolate, had a PFGE profile similar to that of ST646 [SLV of ST177], which is found in the major Chile multiresistant type 19F reference strain) (Fig. 11). ST177 is recorded in the MLST database as coming from type 19F isolates recovered in Spain (associated with meningitis) and Portugal.

A single trimethoprim-sulfamethoxazole-resistant, intermediately penicillin-resistant serotype $19 \mathrm{~F}$ representative of CC156 was detected (ST644). ST644 was also found within serotype 6B (Tables 3 and 4 and Fig. 11). ST43 (from a tetracycline-resistant study isolate and recorded at the MLST database as coming from a $19 \mathrm{~F}$ isolate causing meningitis in the United Kingdom) and ST423 (a lineage 2 isolate also independently discovered in a recent United Kingdom carriage isolate) were also detected in single isolates.

(xix) Serotype 22F. Thirty-seven serotype $22 \mathrm{~F}$ isolates were analyzed, six of which were from pediatric patients. All but one isolate was predicted to be ST433 on the basis of MLST of six isolates and the observation of related PFGE profiles (Fig. 12). ST433 was also independently discovered and associated with a carriage isolate recently recovered in England (see www.mlst .net).

(xx) Serotype 23F. Forty-six (71.9\%) of the 64 serotype $23 \mathrm{~F}$ pediatric isolates were predicted to be within CC37, consisting of ST37 (ancestral type); SLVs ST33, ST626 (characterized by new allele $d d l-83$ ), and ST627; and ST37 DLVs ST628 (new allele $d d l$-95), ST629, and ST625 (Fig. 12 and Table 3). More than half of these lineage 4 type $23 \mathrm{~F}$ isolates were penicillin resistant (Table 2). Eleven multiresistant isolates were presumed to be in CC81 (ST81) (Table 3) on the basis of PFGE and resistance profiles related to reference strain Spain ${ }^{23 F}-1$ (Fig. 12). A set of four ST242 isolates (three of which were sequence typed) were identified that displayed PFGE and resistance profiles highly related to multiresistant, invasive reference strain Taiwan ${ }^{23 \mathrm{~F}}-15$ (30). A single isolate with a divergent PFGE profile was determined to have new allelic profile ST661, within CC662, which is composed primarily of serotype $33 \mathrm{~F}$ and $18 \mathrm{C}$ isolates (Table 3 ). (xxi) Serotype 33F. All seven serotype 33F pediatric isolates were predicted to be within CC662 (ST100, which was associated with meningitis in Spain, and DLV ST636) (Fig. 13). CC662 was common to several serotype 18C (ST496) isolates and a single $23 \mathrm{~F}$ isolate (Table 3 ).

(xxii) Serotype 35B. The 16 serotype $35 \mathrm{~B}$ isolates were divided into two newly discovered STs that were divided into two corresponding PFGE clusters (Fig. 13). All 12 penicillin-nonsusceptible isolates, including one pediatric isolate, were ST451, which was characterized by the new recP44, xpt-77, and $d d l-82$ (type B) alleles. This clone has been discovered among invasive U.S. isolates recovered during other years prior and subsequent to 1999 and is further described elsewhere (1). It is interesting that SLVs of ST451 from type 35B penicillin-resistant isolates have appeared at the MLST database. One of these is additionally documented within a serotype 14 isolate. The four penicillin-sensitive isolates were predicted to be ST452, which has also been detected among U.S. invasive isolates during other years (1).

(xxiii) Serotype 38. Four of the five serotype 38 pediatric isolates were predicted to have in common unique ST393 (Fig. 13), which was also discovered independently in at least one recent type 38 United Kingdom carriage isolate (www.mlst.net).

\section{DISCUSSION}

The purpose of this work was to identify the major CCs within a large number of clinically relevant, individual targeted serotypes and to detect CCs that are common to multiple serotypes. During this process, we also detected and identified rarely occurring genotypes within individual serotypes. It was not the purpose of this study to compare PFGE results to MLST results; however, we used PFGE to select representative isolates to subject to MLST to allow more precise assignments of genetic types and comparisons with results documented in the MLST database. We feel that the analysis presented here will greatly assist us in detecting changes in the genetic structure within individual serotypes of isolate sets from subsequent years and will assist us in expanded molecular surveillance of non-PCV7 serotypes in the year 2001 and beyond.

A second, unanticipated, purpose of this analysis was the use of MLST to detect serotyping errors. Although there were relatively few instances within most serogroups, we did uncover a major error involving a large number of year 1999 serotype $9 \mathrm{~A}$ and $9 \mathrm{~N}$ isolates that were actually found to be type $9 \mathrm{~V}$. We were alerted to this after first noting that several of these type 9A and 9N isolates were ST156 (Spain ${ }^{9 v}-3$ type strain) or ST156 derivatives and after noting that ST156 has never been found previously within types $9 \mathrm{~A}$ and $9 \mathrm{~N}$.

The increasing characterization of pneumococci collected worldwide through the use of MLST became increasingly evident to us during this study. For example, four STs (now designated ST376, ST460, ST473, and ST395) appeared to represent more than $60 \%$ of the serotype $6 \mathrm{~A}$ isolates and were discovered during our work prior to their appearance at www .mlst.net. Several other, similar, instances were observed during the course of this project. We discovered a penicillinresistant type $35 \mathrm{~B}$ clone disseminated across the United States that carried three new MLST alleles that we submitted to the 
MLST database. Within a few months, an SLV was reported at the MLST database as being from Sweden and two SLVs have appeared in other areas within the United States. These observations reflect the fact that, through the use of MLST, there is effective documentation of a high percentage of circulating clonal types. This nonsubjective electronic transfer of precise genetic information is a necessary first step in understanding clone-specific genetic traits differentially affecting the epidemiology and virulence of various globally disseminated strains. The addition of 49 new STs found during this work, combined with 618 STs already recorded at www.mlst.net, brought the total number of known STs to 667 as of 27 November 2002.

It is known that multiple penicillin-resistant strains within individual serotypes arose during the past 25 years through steps requiring multiple horizontal DNA transfer events and point mutations. It is also known that there are multiple existing examples of strains within indistinguishable genotypes expressing distinct serotypes that appear to have arisen through a simpler mechanism of horizontal transfer requiring a single double-crossover event. Such strains have only been examined extensively within PCV7 serotypes. It is plausible that successful clones currently targeted by potent vaccines could gradually shift to alternate nontargeted serotypes through genetic transfer events, analogous to the major shift in the frequency of individual successful penicillin-resistant strains that occurred during the past 25 years. For example, a penicillin-nonsusceptible type $24 \mathrm{~F}$ strain has recently been characterized within an ST previously only documented in invasive, intermediately penicillin-resistant type 14 isolates (29).

Vaccination tends to cause a shift toward carriage of strains of nonvaccine serotypes. Vaccine trial results indicate that PCV7 will reduce the carriage of vaccine serotype strains $(5,6)$. Among children who were immunized with PCV7, a marked reduction in the number of episodes of acute otitis media occurred; however, the proportion of isolates within nonvaccine serotypes significantly increased (11). The degree to which transformation of present vaccine serotype strains to nonvaccine serotypes will contribute to this shift is unknown. The only means by which to detect such occurrences is precise genetic surveillance. We have provided a broad database of genetic types that will greatly facilitate our future invasive pneumococcal surveillance within the United States. This database will allow us to compare CC (or single ST lineage) and serotype associations from invasive population-based isolate sets from subsequent years (and possibly prior years) to assess the potential selective effects of PCV7.

We and others have found several examples of multiple serotypes sharing the same ST; however, these examples have been primarily limited to involve serogroups $6,9,19,14$, and 23. This is still a reason for concern, since PCV7 only includes types $6 \mathrm{~B}, 9 \mathrm{~V}, 14,19 \mathrm{~F}$, and $23 \mathrm{~F}$, and the potential protective effects of PCV7 against other serotypes included within serogroups (i.e., 6A, 9A, 9N, 19A, and 23A) have not been well studied. It is believed that the common basis for clinical isolates of varied serotypes having the same genotype is horizontal transfer of serotype-specific genes; however, this has only been shown at the molecular level in a limited number of instances involving interserogroup transformation events (3, 4). There are no data indicating that intrachromosomal changes could shift serotypes; however, shifts changing the serotype without changing the serogroup have not been examined at the DNA sequence level. This appears likely to be the case with the documented interconversion of types $15 \mathrm{~B}$ and $15 \mathrm{C}(24,33)$. In the near future, sequence information from more of the capsular biosynthetic operons will help to resolve this issue.

CC199, CC13, CC81, CC37, CC66, CC663, CC662, and ST90 represented sets of isolates with both PCV7 and nonPCV7 serotypes, indicating the possibility that horizontal transfers involving an extensive array of both PCV7 and nonPCV7 serotype-specific genes have occurred in the past (Table 3).

ST199 (15B, 15C, 19A, and 19F) and ST90 (6A and 6B) are two examples of STs shared between study set isolates with PVC7 serotypes and one or more non-PCV7 serotypes. Many more examples exist in the MLST database, including ST66 (9N and 19F) and ST81 (19F, 23F, 19A, 9V, and 14) (Table 3). There were only two examples of STs representing non-PCV7 types outside of PCV7 serogroups in combination with other serotypes, but this may be due to the fact that fewer non-PCV7 serotype isolates were included in this analysis. These included ST199 (15B, 15C, 19A, and 19F) and ST218 (12F and 7F) (CC199 and CC218 in Table 3).

It has been estimated that MLST alleles are changed 10-fold more frequently through recombination than by mutation in pneumococci (12); however, the ranges of ST decay rates among pneumococcal populations in nature are not known. The data shown (Fig. 1 to 13 and Tables 2, 3, and 4) provide a pre-PCV7 baseline for determining ST decay rates and for determining serotype shifts within these CCs and single ST lineages.

In summary, this work is necessary to properly monitor horizontal transfer events resulting in serotype changes within virulent clones and the generation of new antibiotic-resistant strains. Currently, pneumococcal clone expansion (and decay) and the emergence of new strains can only be accurately assessed through a system based on MLST. For future proper vaccine formulation strategies within the United States, it is necessary to put into place the most precise surveillance possible. The present effort has provided a reference strain network that will greatly decrease the effort involved in subsequent large genetic surveillance efforts in the United States.

\section{ACKNOWLEDGMENTS}

This work was supported through the ABCs-Emerging Infections Program (EIP), the CDC National Vaccine Program Office, and the CDC Opportunistic Infections Working Group.

We are very grateful to State Laboratory members for providing the isolates and demographic information concerning the isolate set. We especially thank Carolyn Wright and other CDC ABCs-EIP members for providing isolate information. We greatly appreciate the efforts of Angela Brueggemann in verifying our new allele sequences and providing designations for new alleles and STs, and we thank all of the others who are involved in the maintenance of the global pneumococcal MLST database at www.mlst.net. We thank Brian Spratt for providing unpublished ST data. We are grateful to Delois Jackson and Alma Ruth Franklin in the CDC streptococcal serology laboratory for serotyping and MIC results. We thank James Jorgensen and his laboratory personnel for some of the MIC data and Elizabeth Blanton for her summer of work in the CDC laboratory.

\section{REFERENCES}

1. Beall, B., M. C. McEllistrem, R. E. Gertz, D. J. Boxrud, J. M. Besser, L. H. Harrison, J. H. Jorgenson, and C. G. Whitney. 2002. Emergence of a novel penicillin-nonsusceptible, invasive serotype 35B clone of Streptococcus pneumoniae within the United States. J. Infect. Dis. 186:1118-1122. 
2. Coffey, T. J., C. G. Dowson, M. Daniels, J. Zhou, C. Martin, B. G. Spratt, and J. M. Musser. 1991. Horizontal transfer of multiple penicillin-binding protein genes, and capsular biosynthetic genes, in natural populations of Streptococcus pneumoniae. Mol. Microbiol. 5:2255-2260.

3. Coffey, T. J., M. C. Enright, M. Daniels, J. K. Morona, R. Morona, W. Hryniewicz, J. C. Paton, and B. G. Spratt. 1998. Recombinational exchanges at the capsular polysaccharide biosynthetic locus lead to frequent serotype changes among natural isolates of Streptococcus pneumoniae. Mol. Microbiol. 27:73-83

4. Coffey, T. J., M. Daniels, M. C. Enright, and B. G. Spratt. 1999. Serotype 14 variants of the Spanish penicillin-resistant serotype 9V clone of Streptococcus pneumoniae arose by large recombinational replacements of the cps A-pbp1a region. Microbiology 145:2023-2031.

5. Dagan, R., R. Melamed, O. Zamir, and O. Leroy. 1997. Safety and immunogenicity of tetravalent pneumococcal vaccines containing $6 \mathrm{~B}, 14,19 \mathrm{~F}$ and $23 \mathrm{~F}$ polysaccharides conjugated to either tetanus toxoid or diphtheria toxoid in young infants and their boosterability by native polysaccharide antigens. Pediatr. Infect. Dis. 16:1053-1059.

6. Dagan, R., M. Muallem, R. Melamed, O. Leroy, and P. Yagupsky. 1997. Reduction of pneumococcal nasopharyngeal carriage in early infancy after immunization with tetravalent pneumococcal vaccines conjugated to either tetanus toxoid or diphtheria toxoid. Pediatr. Infect. Dis. J. 16:1060-1064.

7. Doit, C., E. Denamur, B. Picard, P. Geslin, J. Elion, and E. Bingen. 1996. Mechanisms of the spread of penicillin resistance in Streptococcus pneumoniae strains causing meningitis in France. J. Infect. Dis. 174:520-528.

8. Enright, M. C., and B. G. Spratt. 1998. A multilocus sequence typing scheme for Streptococcus pneumoniae: identification of clones associated with serious invasive disease. Microbiology 144:3049-3060.

9. Enright, M. C., and B. G. Spratt. 1999. Extensive variation in the $d d l$ gene of penicillin-resistant Streptococcus pneumoniae results from a hitchhiking ef fect driven by the penicillin binding protein $2 b$ gene. Mol. Biol. Evol. 16: 1687-1695.

10. Enright, M. C., D. A. Robinson, G. Randle, E. J. Feil, H. Grundmann, and B. G. Spratt. 2002. The evolutionary history of methicillin-resistant Staphylococcus aureus (MRSA). Proc. Natl. Acad. Sci. USA 99:7687-7692.

11. Eskola, J., T. Kilpi, A. Palmu, J. Jokinen, J. Haapakoski, E. Herva, A Takala, H. Kayhty, P. Karma, R. Kohberger, G. Siber, and P. H. Makela. 2001. Efficacy of a pneumococcal conjugate vaccine against acute otitis media. N. Engl. J. Med. 344:403-409.

12. Feil, E. J., J. M. Smith, M. C. Enright, and B. G. Spratt. 2000. Estimating recombinational parameters in Streptococcus pneumoniae from multilocus sequence typing data. Genetics 154:1439-1450.

13. Feil, E. J., E. C. Holmes, D. E. Bessen, M. S. Chan, N. P. Day, M. C. Enright, R. Goldstein, D. W. Hood, A. Kalia, C. E. Moore, J. Zhou, and B. G. Spratt 2001. Recombination within natural populations of pathogenic bacteria: short-term empirical estimates and long-term phylogenetic consequences. Proc. Natl. Acad. Sci. USA 98:182-187.

14. Figueiredo, A. M., R. Austrian, P. Urbaskova, L. A. Teixeira, and A. Tomasz 1995. Novel penicillin-resistant clones of Streptococcus pneumoniae in the Czech Republic and in Slovakia. Microb. Drug. Resist. 1:71-78.

15. Gherardi, G., J. S. Inostrozo, M. O'Ryan, V. Prado, S. Prieto, C. Arellano, R. R. Facklam, and B. Beall. 1999. A genotypic survey of recent $\beta$-lactamresistant pneumococcal nasopharyngeal isolates from asymptomatic children in Chile. J. Clin. Microbiol. 37:3725-3730.

16. Gherardi, G., C. G. Whitney, R. R. Facklam, and B. Beall. 2000. Major related sets of antibiotic-resistant pneumococci in the United States based on pulsed field gel electrophoresis and $p b p 1 a-p b p 2 b-p b p 2 x-d h f$ restriction profiles. J. Infect. Dis. 181:216-229.

17. Hall, L. M., R. A. Whiley, B. Duke, R. C. George, and A. Efstratiou. 1996 Genetic relatedness within and between serotypes of pneumococci from the United Kingdom: analysis of multilocus enzyme electrophoresis, pulsed field gel electrophoresis, and antimicrobial resistance patterns. J. Clin. Microbiol 34:853-859.

18. Lefèvre, J. C., M. A. Bertrand, and G. Faucon. 1995. Molecular analysis by pulsed-field gel electrophoresis of penicillin-resistant Streptococcus pneumoniae from Toulouse, France. Eur. J. Clin. Microbiol. Infect. Dis. 14:491 497.

19. Martin, M., J. H. Turco, M. E. Zegans, R. R. Facklam, S. Sodha, J. A. Elliott, J. H. Pryor, B. Beall, D. D. Erdman, Y. Y. Baumgartner, P. A. Sanchez, J. D. Schwartzman, J. Montero, A. Schuchat, and C. G. Whitney. 2003. Outbreak of conjunctivitis due to atypical Streptococcus pneumoniae. N. Engl. J. Med. 348:1112-1121.

20. Marton, A., M. Gulyas, R. Munoz, and A. Tomasz. 1991. Extremely high incidence of antibiotic resistance in clinical isolates of Streptococcus pneumoniae in Hungary. J. Infect. Dis. 163:542-548.

21. McDougal, L. K., J. K. Rasheed, J. W. Biddle, and F. C. Tenover. 1995. Identification of multiple clones of extended-spectrum cephalosporin-resistant Streptococcus pneumoniae isolates in the United States. Antimicrob. Agents Chemother. 39:2282-2288.

22. McEllistrem, M. C., M. Pass, J. A. Elliott, C. G. Whitney, and L. H. Harrison. 2000. Clonal groups of penicillin-nonsusceptible Streptococcus pneumoniae in Baltimore, Maryland: a population-based, molecular epidemiologic study. J. Clin. Microbiol. 38:4367-4372.

23. McGee, L., L. McDougal, J. Zhou, B. G. Spratt, F. C. Tenover, R. George, R. Hakenbeck, W. Hryniewicz, J. C. Lefévre, A. Tomasz, and K. P. Klugman. 2001. Nomenclature of major antimicrobial-resistant clones of Streptococcus pneumoniae defined by the Pneumococcal Molecular Epidemiology Network. J. Clin. Microbiol. 39:2565-2571.

24. Meats, E., A. B. Brueggemann, M. C. Enright, K. Sleeman, D. T. Griffiths, D. W. Crook, and B. G. Spratt. 2003. Stability of serotypes during nasopharyngeal carriage of Streptococcus pneumoniae. J. Clin. Microbiol. 41:386-392.

25. Moissenet, D., M. Valcin, V. Marchand, E.-N. Garabedian, P. Geslin, A Garbarg-Chenon, and H. Vu-Thien. 1997. Molecular epidemiology of Streptococcus pneumoniae with decreased susceptibility to penicillin in a Paris children's hospital. J. Clin. Microbiol. 35:298-301.

26. Munoz, R., T. J. Coffey, M. Daniels, C. G. Dowson, G. Laible, J. Casal, R. Hakenbeck, M. Jacobs, J. M. Musser, B. G. Spratt, and A. Tomasz. 1991. Intercontinental spread of a multiresistant clone of serotype $23 \mathrm{~F}$ Streptococcus pneumoniae. J. Infect. Dis. 164:302-306.

27. Munoz, R., J. M. Musser, M. Crain, D. E. Briles, A. Marton, A. J. Parkinson, U. Sorensen, and A. Tomasz. 1992. Geographic distribution of penicillinresistant clones of Streptococcus pneumoniae: characterization by penicillinbinding protein profile, surface protein A typing, and multilocus enzyme analysis. Clin. Infect. Dis. 15:112-118

28. Overweg, K., P. W. Hermans, K. Trzcinski, M. Sluijter, R. de Groot, and W. Hryniewicz. 1999. Multidrug-resistant Streptococcus pneumoniae in Poland: identification of emerging clones. J. Clin. Microbiol. 37:1739-1745.

29. Pantosti, A., G. Gherardi, M. Conte, F. Faella, G. Dicuonzo, and B. Beall. 2002. A novel multidrug-resistant serotype $24 \mathrm{~F}$ Streptococcus pneumoniae strain causing meningitis in Naples, Italy. Clin. Infect. Dis. 35:205-208

30. Shi, Z. Y., M. C. Enright, P. Wilkinson, D. Griffiths, and B. G. Spratt. 1998. Identification of three major clones of multiply antibiotic-resistant Streptococcus pneumoniae in Taiwanese hospitals by multilocus sequence typing. J. Clin. Microbiol. 36:3514-3519.

31. Sibold, C., J. Wang, J. Henrichsen, and R. Hakenbeck. 1992. Genetic relationships of penicillin-susceptible and -resistant Streptococcus pneumoniae strains isolated on different continents. Infect. Immun. 60:4119-4126.

32. Smith, A. M., and K. P. Klugman. 1997. Three predominant clones identified within penicillin-resistant South African isolates of Streptococcus pneumoniae. Microb. Drug. Resist. 3:385-389.

33. Venkateswaran, P. S., N. Stanton, and R. Austrian. 1983. Type variation of strains of Streptococcus pneumoniae in capsular serogroup 15. J. Infect. Dis. 147:1041-1054.

34. Whitney, C. G., M. M. Farley, J. Hadler, L. H. Harrison, C. Lexau, A. Reingold, L. Lefkowitz, P. R. Cieslak, M. Cetron, E. R. Zell, J. H. Jorgensen, and A. Schuchat. 2000. Increasing prevalence of multidrug-resistant Streptococcus pneumoniae in the United States. N. Engl. J. Med. 343:1917-1924. 